\title{
Der »Arabische Frühling« in vergleichender Revolutionsanalyse
}

Ursachen, Verlauf und bisherige Ergebnisse des »Arabischen Frühlings « werden in diesem Beitrag aus der Sicht der vergleichenden Revolutionsanalyse erfasst. Revolutionäre Situationen in Gestalt vielfältiger Konfliktformen sind von revolutionären Ergebnissen zu unterscheiden. Auch die Entwicklungsmöglichkeiten unterschiedlicherpolitischer Systeme, so diesehrbeschränkten einer»sultanistischen «Herrschaft, bleiben zu bedenken. Diese ist ausschließlich aufden Herrscherund seine Vertrauten ausgerichtet, ohne Einrichten nachhaltiger Institutionen, die einen reibungsloseren politischen Wandelermöglichen und die Sicherstellungöffentlicher Güterwieineiner Demokratie gewährleisten. Zahlreiche andere theoretische Erklärungsbausteine sindheranzuziehen, wennes um die Einschätzungder Transformation(schancen) und mögliche Szenarien geht. Dabei gehören der Bevölkerungsdruck durch eine mehrheitlich weitgehend un- oder unterbeschäftigte junge Bevölkerung ebenso zu den Belastungen wie das Fehlen einer regionalen Ordnungsmacht. Hinzu kommenfast überall nach haltige religiös-kulturelle Spannungslinien. Die arabischen Königtümer von Marokko bis Saudi-Arabien haben mit kleineren politischen Öffnungen und Wohlfahrtszahlungen den Protestdruck lindern und ein Scheitern wie das der deutlich sultanistisch ausgerichteten Regime in Libyen und Syrien vermeiden können.

Schlagworte: Politische Gewalt, Rebellion, Sultanismus, Politische Herrschaft, vergleichende Revolutionsanalyse

\section{Einleitung}

Ist nach den gewaltsamen anti-westlichen Protesten in der Septembermitte $2012^{1}$ in Libyen, Ägypten, Jemen, Tunesien, dem Sudan und nahezu in der gesamten muslimischen Welt, nach Protesten, die sich nicht nur gegen ein blasphemisches, eine fremde Religion verachtendes Billigfilmchen ${ }^{2}$ aus den USA, sondern auch gegen die Werte freier Meinungsäußerung richten, der so überraschende »Arabische Früh-

1 Eine erste Fassung dieses Beitrags wurde auf Einladung der ZEIT-Stiftung am 25.09.2012 vorgetragen. Für kritische Kommentare danke ich Guido Mehlkop und den Gutachtern dieser Zeitschrift.

2 Horst Bredekamp (2012) spricht von einem »Bilderweltbürgerkrieg« und der Notwendigkeit, die »substitutive Praxis, die Gleichsetzung von Bild und Körper, zu durchkreuzen«. Die Kunstfreiheit sei im deutschen Grundgesetz z. B. noch mehr geschützt als die Meinungsfreiheit. 
ling « bereits in einen »Winter« übergegangen (Barber 2012), ohne je eine Hochblüte und dann auch Ernte einfahren zu können? ${ }^{3}$

Man merkt unwillkürlich, dass die Analogie zum Naturkreislauf in die Irre führt. Auch handelt es sich hier um andere Protestgruppen als diejenigen des »Arabischen Frühlings «, nämlich um durch religiösen Fanatismus aufgehetzte Elemente des SubProletariats und nicht um die erstaunliche »Mitte «, die sich in den revolutionären Herausforderungen zusammenfand, in Herausforderungen, die nicht religiös geprägt und nicht gegen den Westen oder seine Werte, nicht anti-zionistisch oder antiimperialistisch gerichtet waren. Dies erstaunt umso mehr, als der Westen im Sinne zynischer - andere würden sagen: realistischer - Außenpolitik eher an stabilen Verhältnissen und dem Zugang zu den Rohstoffen als an demokratischer Entwicklung interessiert erscheint. $»$ Wenn es schon ein Autokrat ist, dann ist er wenigstens auf unserer Seite « - so hieß es in den 1960er Jahren mit Blick auf Lateinamerika und lange danach auf Nahost, auch angesichts des Schutzbedarfs für Israel. Andererseits wird in Zukunft verstärkt gelten, dass diese Regionen ihre Menschen aus Verzweiflung nach Europa schicken werden, wenn stattdessen nicht ihre Produkte nachgefragt werden. Der in der englischen Sprache populäre Begriff der "Arabellion« meidet »Klima«- und Jahreszeitanalogien und sagt auch nichts über das Ergebnis dieser Rebellionen aus, wie es in dem Begriff der »arabischen Revolution« ausgedrückt wird. Er kombiniert lediglich den geographischen Ort mit einer korrekten morphologischen Analyse ${ }^{4}$ systemherausfordernder Konfliktformen. ${ }^{5}$

Der Begriff der Rebellion gegen bestehende Herrschaftsformen umgreift zahlreiche Varianten der (gewaltsamen) Herausforderung (Zimmermann 2012: 867-868) wie Demonstrationen, Unruhen, Meutereien, Massenproteste, Revolten und Überlaufen repressiver Kräfte, Plünderungen und terroristische Attacken, vor

3 Andere, wie z. B. der tunesische Präsident Moncef Marzouki (2012), erwähnen sogar einen »Islamist Fall« und einen »Salafi Winter«. Zugleich macht er darauf aufmerksam, dass auf die jüngste Veröffentlichung von Mohammed-Karikaturen in Frankreich keine (gewaltsame) Reaktion erfolgt ist. Auch sind trotz mancher Entwicklungen in diese Richtung der »Islamist Fall« und der »Salafi Winter« nicht eingetreten.

4 Wie notwendig eine begriffliche und analytische Präzision in diesen vergleichenden Analysen ist, wird auch deutlich im Falle der vielbeschworenen »friedlichen deutschen Revolution « von 1989, die morphologisch korrekter als Kombination einer entglittenen Reform von oben durch Michail Gorbatschow, gepaart mit Rebellionen und der Diffusion von Protestmustern in den Satrapiestaaten, letztlich als Abwicklung des letzten großen Kolonialreichs einzuordnen ist (Zimmermann 2003).

5 Wir verwenden den Begriff des »Arabischen Frühlings « eingedenk der angedeuteten Fehlanalogien in Anführungszeichen, um uns dem vorherrschenden Sprachgebrauch nicht zu entziehen. Der nüchternere Begriff der »Arabellion« ist breiter und analytisch zutreffender. Er wird hier gleichermaßen durchgängig verwandt. Spräche man nur von Arabellion, würde ein Kunstbegriff in penetranter Weise in die deutsche Sprache eingeführt. Analytisch völlig falsch ist die Rede von einer arabischen Revolution. 
allem aber die Bereitschaft, sich stärker gegen die Fortführung der bisherigen Herrschaftsordnung auszusprechen, dies notfalls auch organisiert und durch Einsatz von Gewalt. Die jeweilige Form des Protestes und der Rebellion ist einer der Bestimmungsgründe nicht nur des Konfliktverlaufs, sondern letztlich auch seiner Ergebnisse und Folgen. In der vergleichenden Konfliktforschung geht der Begriff der Rebellion und des inneren Krieges einher mit stärkerer Organisierung und gewaltsam vorgetragenen Herausforderungen (Zimmermann 2012). Reine Proteste und Demonstrationen sind dagegen weniger durchorganisiert und nicht auf den Einsatz von Gewalt ausgerichtet. Gewaltorientierte Teilgruppen verfolgen in solchen Massenprotesten oft ihre eigenen Ziele. Es ist wegen der Herausforderung des staatlichen Gewaltmonopols und entsprechender Repressionsfolgen aber anzuraten, beide Protestformen (in der Terminologie der bisherigen Forschung: Protestdemonstrationen und inneren Krieg; Hibbs 1973) auseinanderzuhalten.

Es geht also um eine klare morphologische Betrachtung und die Herausarbeitung von differentia specifica in zweierlei Hinsicht, zum einen mit Blick auf die herkömmliche vergleichende Revolutionsanalyse und zum anderen mit Blick auf die unterschiedlichen Voraussetzungen, Abläufe und bisherigen Ergebnisse der unterschiedlichen Konfliktformen in den arabischen Ländern mit ihren unterschiedlichen Regimeformen. Schließlich wird sich herausstellen, dass ein knappes Dutzend anderer theoretischer Erklärungsansätze heranzuziehen ist, wenn es um die Einschätzung der Transformation(schancen) und möglicher Szenarien geht.

Die hier vorgelegte vergleichende Analyse orientiert sich in starkem Maße an der von Samuel Huntington (1968) aufgeworfenen Fragestellung nach der politischen Ordnung in sich wandelnden Gesellschaften. Dabei suchen wir reduktionistischer als Huntington zu argumentieren, bei dem sich viele theoretische Anregungen finden. Zwar wird hier auf verschiedene Elemente aus der Analyse von Huntington zurückgegriffen, so die Ideen unzureichender politischer Institutionalisierung bei überhöhter sozialer Mobilisierung und die des direkten Eingreifens sozialer Kräfte in die Politik. Die hier gewählte Sichtweise konzentriert sich aber auf Erkenntnisse der vergleichenden Revolutionsanalyse, erweitert um theoretische Einsichten aus den Forschungen zur Transformation politischer Herrschafts- und Systemformen (Merkel 2010). Sie leistet damit einen vorläufigen Beitrag zur Einschätzung und Erklärung der Varianten der »Arabellion«.

Daneben wird die Analyse durch Rückgriffe auf grundlegende Erklärungen für das Verständnis stabiler Herrschaft und ihrer Folgen erweitert. Kern bleibt die Einbeziehung der vergleichenden Revolutionsanalyse in die ansonsten stärker dominierende Regimetransformationslehre. Bei letzterer geht es im Wesentlichen um Veränderungen im politischen Bereich. Die Revolutionsanalyse bezieht dagegen 
Veränderungen in der Sozialstruktur, in den Wertemustern, in den grundsätzlichen Werten und institutionellen Vereinbarungen von Gesellschaften mit ein. Sie umgreift damit die Transformationsanalyse, sieht sich aber angesichts geringer Fallzahlen erfolgreicher Revolutionen auch größeren Schwierigkeiten bei der statistischen Analyse ausgesetzt, also bei dem Versuch, Veränderungen in den abhängigen Variablen kontrolliert auf jeweilige unabhängige Variablen zurückzuführen. Aber auch die Regimetransformationslehre ist auf Grund der Pfadabhängigkeit von Herrschaftsformen in den meisten Fällen als überdeterminiert zu bezeichnen: $\mathrm{Zu}$ viele plausible Erklärungen können gleichzeitig angeboten werden, ohne dass der Einfluss der jeweiligen Faktoren hinreichend kontrolliert werden kann. Dies gilt jedenfalls, je mehr man den Rahmen der Analyse zeitlich erweitert.

Nachfolgend (Abschnitt 2) werden die verschiedenen Stufen der Entscheidungsherausforderung von Krisen bis zu erfolgreichen Revolutionen und damit verbundene wichtige analytische Unterscheidungen anhand zahlreicher historischer Fälle kurz herausgearbeitet. Gleiches gilt für die fünf zentralen Erklärungsmomente für den Ausbruch und die erfolgreiche Durchsetzung revolutionärer Forderungen. Der zweite grundlegende Erklärungsstrang wird aus der Differentialdiagnose politischer Herrschaftsformen, ihrer konstitutiven Elemente, ihres Fortdauerns und der damit verbundenen Entwicklungschancen zu einer demokratischen Herrschaftsform gewonnen.

Die Ausführungen über die außergewöhnliche Entstehung des »Arabischen Frühlings « und die besondere Motivation und Unterschiede zu großen Revolutionsführern der Vergangenheit (Abschnitt 3) berühren einige der Überraschungen und Neuheiten dieser Proteste und Rebellionen, zeigen andererseits aber auch historische »Parallelen« auf.

In den vorliegenden Fällen der Rebellion stehen dabei letztlich zwei Herrschaftsformen im Vordergrund: der Sultanismus ${ }^{6}$ als personalisierte und institutionell wenig differenzierte Herrschaftsform, deutlich unterschieden von Formen traditionaler monarchischer Herrschaft mit stärkerer Rücksichtnahme auf Belange der Bevölkerung und damit auch größerer Legitimität. Wie dabei in der Differentialdiagnose des »Arabischen Frühlings« aus der Sicht der vergleichenden Revolutionsanalyse deutlich wird (Abschnitt 4), können letztere Regimeformen sich aber ebenfalls nachhaltigen Rebellionen mit ungewissem Ausgang gegenübersehen. Damit geraten Fragen der internationalen Politik und Sicherheit in den Blick, die hier nur am

6 Max Weber (1964: 171) betont idealtypisch die »traditionsungebundene Willkür« dieser Form der Patrimonialherrschaft, die ins Extrem entwickelte »Sphäre der freien Willkür und Gnade« und damit das Fehlen von »jeder Form rationaler Herrschaft«. 
Rande berührt werden können, aber in den angedeuteten Optionen (Abschnitt 5) und zukünftigen Szenarien (Abschnitt 6) aufscheinen.

\section{Revolutionen und Regimetransformation: Erklärungselemente und Befunde}

Vier Phasen der Herausforderung sind zu unterscheiden: Krisen, große Systemkrisen, revolutionäre Situationen und schließlich Revolutionen als Ergebnis grundlegender gesellschaftlicher und politischer Veränderungen. Krisen sind im Sinne des griechischen Ursprungs des Wortes Entscheidungsherausforderungen für die Herrschenden. Diese Entscheidungsherausforderungen sind häufiger als große Systemkrisen, wie die Weltwirtschaftskrise der 1930er Jahre, in denen die bisherige Herrschaftsordnung auf die Probe gestellt wird. Noch deutlicher wird diese Herausforderung in den noch selteneren revolutionären Situationen, in denen mehr oder weniger zentrale Gruppen zugleich das bisherige Herrschaftsmodell und das Modell des Wirtschaftens aufkünden (wie im kurzen und »heißen« Mai 1968 in Frankreich oder in vielen Ländern nach der Niederlage im Ersten Weltkrieg). Schließlich landet man bei den zwei Dutzend und wenig mehr erfolgreichen Revolutionen seit Beginn der Neuzeit. Wie der deutsche Satiriker Heinrich Hoffmann, der vom »Struwwelpeter«, 1848 schon treffend wusste: »Revolution. Ein auf dem Markte der Welthändel sehr gesuchter, aber auch sehr theuerer und kostspieliger Artikel« (Struwwel 1848: 33).

Die drei wesentlichen Ausgänge einer revolutionären Situation sind also das Durchsetzen der bislang Herrschenden durch Repression wie im Iran im Jahre 2011, das Austragen der Auseinandersetzung wie momentan in Syrien in einem blutigen Bürgerkrieg, der das Gewaltausmaß einer Revolution meist deutlich übertrifft, oder der Sieg der Herausforderer und die Umsetzung ihres revolutionären Programms. Als inhaltlich offene, nur an strukturellen Änderungen orientierte Definition bietet sich somit an:

»Als Revolution ist der erfolgreiche Umsturz der bisher herrschenden Elite(n) durch eine neue Elite (neue Eliten) zu verstehen, die nach ihrer (meist mit größerem Gewaltgebrauch ${ }^{7}$ und der Mobilisierung von Massen verbundenen) Machtübernahme die Sozialstruktur und damit auch die Herrschaftsstruktur fundamental verändert (verändern)« (Zimmermann 1981: 142).

7 Die Gewalt nimmt jedoch eher nach einer Revolution zu, weil Gegner noch endgültig bezwungen werden müssen oder Rechnungen offen sind, weil sich die Lager aufspalten oder die Sezession eines Landesteils droht. 
Lenkt man den Blick auf die Veränderung der politischen Herrschaft, so sind drei Phasen mit jeweils typischen Herausforderungen zu unterscheiden: In der ersten Phase bricht die alte Herrschaft zusammen. Das Gewalt- und oft auch Meinungsmonopol wird herausgefordert. Die beiden Extremfälle sind dann entweder eine erneute Stabilisierung des bisherigen Regimes oder Anarchie.

In der zweiten Phase des Regimeübergangs entscheidet sich, wie viel von der alten Herrschaftsform und dem jeweiligen »Konsens « und wie viele von den AltEliten diesen Übergang überleben. Beide, Alt-Eliten und Neu-Eliten, trauen sich gegenseitig nicht, müssen aber Möglichkeiten des Aushandelns finden, soll nicht die reine Gewalt, wie in einem Bürgerkrieg, entscheiden. Spanien in der Übergangsphase nach Francisco Francos Tod 1975 liefert hier ein Meisterbeispiel (Colomer 2000) der möglichen und dann sehr erfolgreich genutzten Übergangschancen zu einer konsolidierten Demokratie.

Damit ist bereits die dritte Phase benannt, in der eine neue politische und oft zugleich wirtschaftliche Organisationsform geschaffen werden soll. Handelt es sich um den Wandel zur freiheitlichen Demokratie, so dauert diese Phase leicht ein Jahrzehnt, wenn man von dem Plan zu einer verfassungsgebenden Versammlung bis zur ersten freien Wahl rechnet; mehr als ein Jahrzehnt, wenn man die demokratische Konsolidierung mit dem Übergang der Macht an die bisherige Opposition ansetzt; oft zwei Generationen, wenn man von einem erneuten friedlichen Machtwechsel (Huntington 1991) als Merkmal der Konsolidierung ausgeht. Ist letztere erreicht, wird damit auch der besondere Charakter einer demokratischen Ordnung deutlich, nämlich in friedlicher, freier und geheimer Wahl unterliegen zu können, aber mit dem Recht und der Chance, beim nächsten Mal unbehindert den Sieg davonzutragen. Der friedliche Machtwechsel in Demokratien schützt also nicht nur die Herrschaftsordnung, sondern auch die Herrschenden.

Eine der großen Schwierigkeiten in den Phasen zwei und drei besteht darin, die Teilnahme der Massen auf die institutionell vorgegebenen Kanäle zu richten bzw. solche schnell bereitzustellen. Das Aushandeln der neuen Herrschaftsform geschieht in den erfolgreichen Fällen unter den Alt- und Neu-Eliten, die dann die Legitimierung durch die Wähler oder die jeweiligen Bevölkerungsgruppen gewinnen müssen, für die sie zu sprechen behaupten.

Sobald Massendemonstrationen, Gewalt und andere Herausforderungen auftreten, ist der Spielraum für kontrolliertes politisches Handeln und Konsens weiter verringert. An dieser Stelle werden die politische Geschicklichkeit der Führer und die Kunst des Aushandelns besonders wichtig. Ein weiterer Indikator ist, ob und wieweit die politischen Führer für ihre Klientel sprechen, in der Lage sind, diese ruhig zu stellen, und sie von einer direkten Teilnahme an den Verhandlungstischen 
fernzuhalten (wie in Spanien 1975). Häufig gelingt dies den politischen Führern nicht, oder sie treiben die Massen sogar an (wie zu Beginn der politischen Transformation in Portugal 1974; Linz/Stepan 1996: 126). Folglich ähnelt diese Phase oft einer Situation der »Doppelherrschaft« (Trotzki 1967) oder »multipler Souveränität« (Tilly 1975: 522).

Pakte zwischen Eliten in dieser Phase unterscheiden sich nach Reichweite, bindender Wirkung, ihrer zeitlichen Einbettung und ihrer Nachhaltigkeit. In der Konsolidierungsphase ist zwischen Bestands- und Flussgrößen zu unterscheiden. Erstere bezeichnen grundlegende institutionelle Regelungen (North 1992). Manchmal entwickeln sich diese neuen Institutionen, indem aus Strömungsgrößen Bestand wird. Im Allgemeinen besitzen die neuen Regeln der Privatisierung, der Eigentumsrechte, der politischen Interessensartikulation und der wirtschaftlichen Akkumulation Bestandscharakter und erzeugen damit Verhaltensmuster des Akzeptierens, der Zurückweisung oder der Vermeidung.

Diese analytischen und grundsätzlichen Überlegungen zu den Phasen des Regimeübergangs bedürfen der Ergänzung, wenn es um die Chancen einer erfolgreichen Regimetransformation geht. Handelt es sich um den Übergang zu einer Demokratie, so haben Juan Linz und Alfred Stepan (1996) deutlich gemacht, dass die Chancen von früheren Demokratien, über autoritäre Regime, post-totalitäre Regime, totalitäre Regime bis zu den »sultanistischen Regimen « deutlich abnehmen und letztlich gegen Null sinken. In autoritären Regimen (»autoritär« in dieser engeren Sicht) ist lediglich der politische Raum zensiert, in totalitären alle Bereiche der Gesellschaft, mit leichter Lockerung in post-totalitären (Ungarn, Polen). In einer »sultanistischen « Herrschaft bestimmt der »Sultan« (Staatspräsident) allein und unabhängig von Regeln, so dass keinerlei institutionelle Voraussetzungen für einen zügigen Regimewechsel vorliegen (Rumänien unter Nicolae Ceauşescu, Libyen, Syrien). In allen Fällen ist die Entwicklung pfadabhängig: das alte Regime hinterlässt mehr oder wenige deutliche Spuren und beeinträchtigt damit die Erfolgschancen eines neuen (demokratischen) Regimes.

Wendet man die bisherigen Instrumente der Analyse auf die Länder des »Arabischen Frühlings « an, so lautet die Kurzdiagnose: Sultanistische Regime stehen vor revolutionären Herausforderungen mit neuen Eliten, die sich in vielen Fällen noch nicht herausgeschält haben. Letzteres ist ein für Revolutionen typischer Vorgang. Sie erstrecken sich über mehrere Phasen (Französische Revolution 1789, Chinesische Revolution 1911, Russische Revolution 1917), in denen unterschiedlich radikale Forderungen von unterschiedlichen Führern mit unterschiedlicher Überlebensdauer vorgebracht werden. 
Die Ursachen und Bedingungen für erfolgreiche Revolutionen bleiben zu klären. Jack Goldstone (2003; auch Zimmermann 2011; 2006) bildet hier den Konsens der vergleichenden Revolutionsforschung in fünf Faktorgruppen ab: (1) Effizienzkrise der als ungerecht wahrgenommenen staatlichen Autorität; (2) entfremdete Eliten, gespalten im Dissens über den Reformkurs; (3) massive Wohlfahrtsverschlechterung in Stadt und Land; (4) Entstehen einer angriffswilligen elite-geleiteten Volkskoalition und (5) eine zusammenschweißende, Angriffe rechtfertigende und Alternativen aufzeigende Ideologie. Jeder einzelne oder mehrere Faktoren können Einzelformen des Konflikts auslösen, zusammen erzeugen sie eine revolutionäre Situation, das Überlaufen von Sicherheitskräften und Armee zu den Revolutionären gar eine Revolution (Zimmermann 1981:163).

So ergeben sich mit Blick auf die Verhinderung von Revolutionen drei zentrale Sicherungen: Die Herrscher und die Formen der Herrschaft werden als effektiv und gerecht eingeschätzt, die Eliten sind nicht gespalten und verhalten sich loyal zum System, und die breite Bevölkerung erreicht ihr Versorgungsniveau in vorhersagbarer Weise. Nur wenn einer dieser Faktoren einbricht, ergeben sich Chancen für eine revolutionäre Mobilisierung, und die Tür öffnet sich gegenüber zahlreichen Bedingungsfaktoren und damit ausgelösten Ereignisketten. Alle diese erklärenden Momente werden sich in der Deutung des »Arabischen Frühlings « wiederfinden.

\section{Zur Analyse des »Arabischen Frühlings«}

Nachfolgend sei ein Blick auf die außergewöhnliche Entstehung des »Arabischen Frühlings «, auf die besondere Motivation der Akteure und auf Unterschiede zu großen Revolutionsführern der Vergangenheit geworfen. Dieser Abschnitt liefert wichtige deskriptive und theoriegeleitete Informationen zum Verständnis der Vorgänge. Er wird im vierten Teil durch dichtere ländervergleichende Daten erweitert werden.

\subsection{Außergewöhnliche Entstehung}

Huntington hat in seinem bedeutendsten Werk »Political Order in Changing Societies « (1968) Revolutionen in funktionaler Analyse als den Versuch gedeutet, die Bereiche der Politik und der gesellschaftlichen Entwicklung auf »einen« Stand zu bringen, zumeist auf den fortgeschrittenerer Gesellschaften, die als Vergleich dienen (z. B. England für Frankreich im 18. Jahrhundert, Frankreich dann für andere europäische Länder). Die wahrlich hilflose Gesellschaft ist in der Sicht von Huntington diejenige, die noch nicht einmal zu einer Revolution in der Lage ist (wie momentan Nordkorea). Beobachtbar ist, dass die Potentaten der öl- und gasreichen Staaten einen (kleinen) Teil ihrer Gewinne aus Rohstoffen an die einheimische Be- 
völkerung zur Ruhigstellung weiterreichen (vgl. auch das theoretische Modell von Roland Hodler 2013). Ein weiterer Teil geht in die militärische Sicherung, die letztlich von den gelieferten Militärgütern und Sicherheitsgarantien des größten Rohstoffverbrauchers abhängt, den USA. Die Herrscher sind militärisch und als Lieferant von Rohstoffen von der Nachfrage vor allem der USA und Europas abhängig (es sei denn, man wechselte in absehbarer Zeit zu den Chinesen). Zugleich ist der Westen - vor allem die USA wegen ihres Eintretens als Schutzmacht für Israel und gleichermaßen wegen ihres dominanten kulturellen und materiellen Einflusses leicht das Ziel gesteuerter und ungesteuerter Massenattacken.

Wenn diese Analyse stimmt - die Massenunruhen gegen westliche »Provokationen « liefern ein jüngstes Beispiel -, wird die Entstehung der Arabellion in der Tat außergewöhnlich. Unerwartet in ihrer zunächst und zumeist gewaltfrei vorgetragenen Form kamen die Forderungen aus der bürgerlichen Mitte mit dem Ziel, öffentliche Güter wie Freiheit, Chancengleichheit, Meinungsfreiheit, Rechtssicherheit, Schutz der Eigentumsrechte, ein allseits akzeptiertes staatliches Gewaltmonopol, kurzum Demokratie herzustellen (Marzouki 2012; Mursi 2013). Diese Art der öffentlichen Güter ist das Kennzeichen demokratischer Herrschaftsordnung. ${ }^{8}$ Hinzu kamen der weitverbreitete Ärger über Preissteigerungen, die schlechte Versorgungslage und die Korruption der Führungsschichten. Entscheidend ist der Reformwille von innen. ${ }^{9}$

Berücksichtigt man die Ergebnisse und Erklärungen der vergleichenden Demokratisierungsforschung (Weiffen 2009), so ist der vielleicht berühmteste Befund das Starren auf die Grenzmarke von 6.000 US-Dollar Pro-Kopf-Einkommen. In Spanien war dieses Niveau 1975 schon leicht überschritten. Aus Ungarn wird aus den 1980er Jahren von den Oppositionskräften berichtet, dass sie die spanischen Entwicklungen genau verfolgt und ungeduldig auf ein ähnliches Entwicklungsniveau gewartet haben. Vielleicht ist es kein Zufall, dass sich das höchste Pro-Kopf-Einkommen mit

8 Laut Freedom House (2011) wurde im Jahre 2011 kein arabisches Land zu den freien Systemen gerechnet. Marokko, Tunesien, Libanon, Kuwait und Komoren erreichten den Status halbfreier und die übrigen 17 Staaten den unfreier Systeme. Saudi-Arabien rangiert gar in einer Liga mit Nordkorea und Äquatorial-Guinea.

9 Wenngleich, wie im Falle der Bundesrepublik Deutschland nach dem Zweiten Weltkrieg, auch starke externe Auflagen seitens der alliierten Siegermächte oder auch externer Gläubigerstaaten erfolgen können. Dies - etwa harte Auflagen des Internationalen Währungsfonds (IWF) - ist im Zeitalter des Anti-Kolonialismus aber leicht ein Antidot angesichts multipolarer und unklarer Machtstrukturen und kann im Gegenteil zur weiteren Destabilisierung der ökonomischen und politischen Verhältnisse beitragen. 
8.860 US-Dollar ${ }^{10} \mathrm{im}$ Ausgangsland der Arabellion, in Tunesien, findet, wenn man von den rohstoffreichen Ländern absieht.

Seymour Martin Lipset (1994) begründet diesen Veränderungsdruck auf nichtdemokratische politische Systeme, über die Verwendung der Steuern mitzubestimmen (»no taxation without representation «), mit dem gestiegenen beruflichen Professionalismus in diesen Ländern, mit dem Wechselgeflecht von neuen Organisationsstrukturen und Möglichkeiten der Mobilisierung sowie zahlreichen weiteren Faktoren. Allerdings ist Demokratie nicht eine zwingende Folge dieses gestiegenen Entwicklungstandes, sondern, wie Huntington (1984) ebenfalls skeptisch bemerkt hat, nur eine unter den erweiterten politischen Optionen, die bei steigenden Einkommensverhältnissen in den Blick geraten (China in den nächsten Jahren).

Ein zweites Motiv für diese neuen Protestkoalitionen schält sich unmittelbar heraus: die Wahrnehmung globaler Rückständigkeit. Nach der Öffnung zu neuen politischen Herrschaftsformen und der damit eingeleiteten Wirtschaftsdynamik (auch zu Krisen) in Südeuropa, Lateinamerika, dem Ostblock und in Südostasien wird den professionelleren Kreisen deutlich, unterstützt durch ihre Verwandten und Freunde im westlichen Ausland, wie grundlegend politische und wirtschaftliche Freiheiten, ein Eindämmen der Korruption und vor allen Dingen eine im internationalen Wettbewerb konkurrenzfähige Schulbildung werden.

Die sogenannte Selektoratstheorie (Bueno de Mesquita et al. 2003) setzt hier ein. In einem sultanistischen Regime wird die Mehrzahl der Güter für die Clique des Sultans als seine Privatgüter hergestellt oder eingeführt. Diese Güter kann er sich mühelos aneignen. Erweitert sich das Selektorat - das sind die unmittelbaren Mitbestimmer für die Auswahl der Herrscher - hinreichend, geraten auch öffentliche Güter, die im Interesse der gesamten Bevölkerung liegen, in den Blick. Die Mobilisierung breiter Bevölkerungsgruppen ist dann das Ziel neuer demokratischer Führer.

Die dritte Überraschung bestand darin, dass nicht etwa islamistische Terrorgruppen schiitischer oder sunnitischer Art gegen die jeweils andere Form der islamischen Herrschaft revolutionäre Veränderungen eingeleitet haben. Vielmehr hielten sich sowohl die weniger militanten muslimischen Gruppen wie die Muslimbrüder als auch die militanten Terrorverbände bei den Massenprotesten zurück. ${ }^{11}$ Beide spielten z. B. bei der Entmachtung von Hosni Mubarak keine Rolle.

10 Basierend auf Daten der Weltbank (Stand: 2011, Kaufkraftparität in US-Dollar; http://data.worldbank.org/).

11 Die Führung der muslimischen Al-Azhar-Universität in Kairo erklärte die Proteste gar für »haram«, als verboten aus religiösen Gründen. 
Außergewöhnlich ist viertens auch die Beteiligung von Frauen an diesen Massendemonstrationen. Die Rolle der Frauen in diesen Ländern ist durch drei zentrale Herausforderungen gekennzeichnet: Abbau der Hürden für die eigene Schulbildung, damit die Chancen in allen Berufen und die Wahrscheinlichkeit, die eigene Kinderzahl durch andere Optionen und besseres Wissen zu verringern, steigen. Die dritte Herausforderung liegt darin, neue, weniger gewaltsame Formen der Konfliktaustragung in eine männlich dominierte Umwelt zu bringen (wenngleich fanatische Frauen in revolutionären Auseinandersetzungen mitunter eine Rolle gespielt haben und auch massiv in Terrorangriffe eingebunden sind). Letzterer ist vermutlich der schwierigste Aspekt und kommt einer Revolution anderer Art gleich. Die ersteren beiden Aspekte entfalten, wenn auch langsam, ihre eigene Dynamik. Ein entscheidender Grund für die Rückständigkeit der arabischen Welt im globalen Maßstab ist die Nichtnutzung der weiblichen Intelligenz in produktiven Berufen (Landes 1998).

Unerwartet in ihrer Mobilisierungsform und Orchestrierung war fünftens der Ablauf vieler Proteste. (Nahezu) Live-Übertragungen durch Videogeräte und Mobiltelefone sorgten dafür, dass das Leiden der eigenen Opfer sofort und glaubhaft dokumentiert werden konnte, dass neue Formen der Koordination über Microblogging (wie Twitter) entwickelt und dass die Verlautbarungen der Regierung nachhaltig hinterfragt und kritisiert wurden, auch durch Graffiti und Kunstwerke. All dies kam in dieser Wucht und Kombination unerwartet für die Herrschenden, deren Hilfskräfte oft ja Verwandte der Protestler (ähnlich wie in der DDR 1989) waren. Somit wird in doppelter Hinsicht verständlich, wie gering die Kontrollausübung durch Armee und Geheimdienste tatsächlich ausfiel. ${ }^{12}$

Die Informationen über lokale und weitergehende Ärgernisse liefen einerseits über dezentrale private Medienkanäle und parallel über unabhängige ausländische Berichterstattungen. Die Form und in mancher Hinsicht die Geschwindigkeit scheinen das Außergewöhnliche zu sein, sind es aber nicht, wenn man schon an die »Grande Peur« vor einer adligen Konterrevolution in der Französischen Revolution von 1789 (Lefebvre 1970) denkt, eine Furcht, die sich im Rhythmus eines Tagesritts von Paris aus verbreitet hat. Das Jahr 1848 mit der Welle von revolutionären Umsturzversuchen in Europa (mit Ausnahme Großbritanniens) oder die Jahre zu Ende des Ersten Weltkriegs und unmittelbar danach liefern weitere Beispiele für eine starke Diffusion von revolutionären Protestströmungen, wenngleich die wichtigeren Ursachen jeweils im eigenen Land liegen. Neu sind allerdings die mit den neuen elektronischen Medien verbundene Breite der Informierung, deren interaktiver 
Charakter wie vor allem die deutlich erhöhten Chancen einer erfolgreichen Mobilisierung von verschiedenen Bevölkerungsteilen gegen das vorherrschende System.

Außergewöhnlich sind diese Protestkoalitionen schließlich insofern, als die meisten Beobachter eher eine nationalistische anti-koloniale Front gegen den Westen und das Hassobjekt Israel erwartet haben. Die Protestler nehmen jetzt aber die eigene korrupte Führung (weniger ihre ausländischen Verbündeten) in den Blick als Verursacher für die eigene jahrzehntelange Rückständigkeit.

Nimmt man die Lipset-These, die revolutionären Vorbilder aus Südeuropa, Lateinamerika, in jüngerer Zeit aus dem Zerfall des Kommunismus und aus permanenten weltweiten Protesten, kombiniert mit den neuen technischen Möglichkeiten der Selbstorganisation, deutlich erweitert durch ausländische Hilfe, so bleibt allerdings wenig Außergewöhnliches, sondern nur, dass die über Jahrzehnte versagt habenden eigenen Eliten in den Blick geraten, dass keine Externalisierung interner Konflikte geschieht und dass das Leitbild ein offenbar starker Wille nach demokratischen öffentlichen Gütern ist. Dies alles folgt traditionellen westlichen Mustern, allerdings eingebettet in eine friedlich sein sollende Variante muslimischer Religionsausübung, bei den eigentlichen Protestgruppen gar in die Forderung nach einem säkularen Muslimstaat.

Skepsis wird bleiben. So hat Huntington in seinem Werk »Clash of Civilizations « (1996) auf zwei grundlegende strukturelle Verwerfungen der islamischen Welt hingewiesen: auf den weit über 50\% liegenden Anteil junger Menschen unter 30 Jahren mit nahezu keinen oder sehr geringen Chancen auf angemessene Beschäftigung und auf das Fehlen einer regionalen Ordnungsmacht. Diese beiden strukturellen Bedingungen bestehen unvermindert fort. Weder das arme Ägypten noch das reiche Saudi-Arabien, schon gar nicht die superreichen Emirate wie Katar mit seiner aktiven Medienpolitik (Reed 2013), sind zu solcher Ordnungspolitik in der Lage. Gelingt es freilich, die friedliche demokratische Öffnung, auch mit Unterstützung des Westens (Marshallpläne, Investitionen, Ausbildung), nachhaltig zu gestalten, dann wird das vielbeschworene Diktum von den »blutigen Grenzen« des Islams vielleicht widerlegt, werden Zollabbau und Wirtschaftsunion an seine Stelle treten. Letztlich steht hinter dem die tägliche Forderung nach Aufrechterhaltung des Gewaltmonopols nach innen und außen wie z. B. die Entwaffnung der Stammesmilizen. 


\subsection{Besondere Motivation der Akteure und Unterschiede zu großen Revolutionsführern der Vergangenheit ${ }^{13}$}

Nimmt man die radikalen Führer der Französischen Revolution, die der kommunistischen in Russland, China, Kuba und Vietnam allemal, die der nationalsozialistischen Führungseliten und der schiitischen Machthaber im Iran, so sind viele der revolutionären Forderungen auch deutlich gegen die internationale Machtbalance gerichtet. Stephen Walt (1996) und Huntington (1968) sehen Revolutionen als Bedrohung des internationalen militärischen und ideologischen Gleichgewichts, besonders wenn sie mit der stärksten Mobilisierungskraft gepaart sind: revolutionärem Nationalismus. Ähnlich wie beim internationalen Terrorismus (Zimmermann 2009; 2011) findet sich in der Abwehr gegen ausländische Besatzer oder Eindringlinge die größte Protestkoalition, jenseits aller religiösen Spaltungen oder geschlechtlichen Diskriminierung. ${ }^{14}$

Die Anpassungsbemühungen heimischer Herrschaftsstrukturen und des jeweiligen politischen Systems an fortschrittliche und erfolgreiche Staaten sind wettbewerbsverschärfend, da dann neue Konkurrenten am Weltmarkt auftreten. Sie bieten Möglichkeiten der Entwicklung, einer optimaleren globalen Arbeits- und Wissensteilung. Damit kann sich das Kalkül der Herrschaft weg von militärischen Auseinandersetzungen auf die langfristig wechselseitigen Vorteile wirtschaftlicher Verflechtungen richten. Ein anderes Szenario kann dann aber wieder drohen, das des Kreislaufs von wirtschaftlicher Macht zu militärischer Macht, externem Krieg und möglicher Überausdehnung (Kennedy 1989).

Die Theorie und Befunde des demokratischen Friedens (Russett/Oneal 2001; Bussmann et al. 2009) sind hier von Bedeutung: Demokratien untereinander begeben sich nicht in kriegerische Auseinandersetzungen, wozu nicht nur die gemeinsame Staatsform mit friedlicher Konfliktverarbeitung beiträgt, sondern auch der internationale Handel und ebenfalls in abnehmender Stärke die Mitgliedschaft in internationalen Organisationen. Andererseits ist eine Demokratie in einem ansonsten autokratischen Umfeld besonders gefährdet (Mansfield/Snyder 2005), was neben weiteren Konfliktursachen auch auf die Auseinandersetzung mit Israel zutrifft.

13 Diese Analyse ist hier bewusst kurz gehalten. Umfangreicheres Datenmaterial bleibt zu erheben und zu analysieren, und selbst dann werden Fehlschlüsse aus Persönlichkeitsmerkmalen auf historische Abläufe häufig nicht vermieden.

14 Für die Arabellion ist die Gefahr eines nach außen gewandten revolutionären Nationalismus keineswegs undenkbar, erhöht sich doch die Zahl der Mitsprecher in der Auseinandersetzung mit Israel und damit auch die Gefahr populistischer Explosionen. 
Interessanterweise haben die neuen arabischen revolutionären Führer keine sozialistischen Visionen mehr (wie früher Gamal Abdel Nasser), keine Gesamtideologie, auch nicht eine solche eines dritten Weges der Entwicklungsländer (Marzouki 2012; Mursi 2013). Allerdings ist das Bild einer gemäßigt muslimischen freiheitlichen Herrschaft mit klaren Zielen noch kaum erkennbar. Die Rhetorik nach innen und außen ist von klaren rechtlichen Schritten und deren Einhaltung zu unterscheiden.

Fragt man nach Unterschieden in der Persönlichkeitsstruktur der arabischen revolutionären Führer, so ist damit ein weites Feld geöffnet. Die auf Persönlichkeiten zielende vergleichende Revolutionsforschung leidet unter mangelnden Fallzahlen und damit mangelnden Vergleichsmöglichkeiten. Prinzipiell sind spezielle Führungsmerkmale (etwa jetzt die Coup-ähnliche Geschicklichkeit von Mohammed Mursi gegenüber der alten Armeeführung und gegenüber dem Iran) immer im Zusammenhang mit institutionellen Strukturen und situativen Gegebenheiten zu sehen. Man kann also aus Führungsmerkmalen nicht unmittelbar auf revolutionäre Ergebnisse schließen.

Die vergleichende Revolutionsforschung erteilt jedenfalls zwei deutliche Lehren: Erstens müssen revolutionäre Herausforderer nicht nur strategisches Talent besitzen, sondern, fast noch mehr, taktisch begabt sein und aus mehrfachen Niederlagen lernen (Wladimir I. Lenin, Mao Zedong, Fidel Castro, Adolf Hitler). Nicht ganz ohne Grund hat Huntington (1968) in diesem Zusammenhang Karl Marx einen "politischen Primitivling" gescholten und Lenin als taktisches Genie bezeichnet. Zweitens gilt, dass Revolutionen als Ergebnis ein sich herausbildender Prozess sind, der andere Gruppen und ideologische Richtungen nach vorn und an die Spitze bringt. Dies führt dann zu dem von Vilfredo Pareto beschworenen »Kreislauf der Eliten«, freilich keineswegs mit einer regelmäßigen Abfolge einzelner Stufen (Zimmermann [1983] 2011).

Einiges von der Vielfalt der vorstehenden Aspekte lässt sich in der Konzentration auf wenige Erklärungsfaktoren nachfolgend deutlicher herausarbeiten. In aller Kürze handelt es sich um die grundlegenden Befunde, die sich für die Arabellion aus der Sicht der vergleichenden Revolutionsanalyse ergeben.

\section{Differentialdiagnose der Arabellion aus der Sicht der vergleichenden Revolutionsanalyse}

Zunächst werden die schon erwähnten fünf zentralen Erklärungselemente für erfolgreiche Revolutionen aus der summarischen Analyse von Goldstone (2003) auf 
die acht Länder mit Rebellionserfahrungen in jüngster Zeit angewandt. ${ }^{15}$ Dabei werden zwei Gruppen politischer Regime deutlich: traditionale Monarchien in Marokko und Jordanien und auf der arabischen Halbinsel, bei denen einzelne Erklärungsmomente bzw. mehrere gehäuft nicht anzutreffen sind, im Unterschied zu den gestürzten sultanistischen Herrschaftsmodellen in Tunesien, Libyen, Ägypten oder noch (vorübergehend) stabilisierten andernorts. In diese Differentialdiagnose werden einige Daten zum Entwicklungsstand, der ethnographischen und religiösen $\mathrm{Zu}-$ sammensetzung und andere wichtige länderspezifische Informationen eingebunden, stehen aber hier nicht im Zentrum.

Nimmt man die acht arabischen Länder mit Massenprotesten nach 2010 und dem Ruf nach Änderung des politischen Systems - weg von der Dekaden alten autoritären Herrschaft sultanistischen Charakters (Linz/Stepan 1996) wie in Tunesien, Ägypten und Bahrain sowie im Jemen bzw. dynastischer Herrschaft wie in Syrien (Baschar al-Assad und die ihn stützende Gruppe der Alawiten, die lediglich 10\% der Bevölkerung ausmachen) - und unterzieht die dortigen Abläufe und Entwicklungen einer Analyse mittels der von Goldstone (2003) benannten fünf Erklärungsfaktoren, so lassen sich zwei große Ländergruppen unterscheiden: die autoritären Königreiche Marokko und Jordanien auf der einen und die restlichen sechs Länder auf der anderen Seite. Unter den sechs verbleibenden Ländern ergeben sich weitere Differenzierungen. In der Gruppe der autoritären Königreiche haben beginnende Massenproteste zu Reformkonzessionen geführt. ${ }^{16}$ Dabei ist die Herrschaft des Haschemitenkönigs Abdullah - angesichts des Palästinenseranteils von über 50\% an der Bevölkerung und seit dem Bürgerkrieg aus Syrien hereinströmender Flüchtlinge (inzwischen fast eine halbe Million) - als stärker gefährdet zu bezeichnen denn die von König Mohammed VI. in Marokko. In beiden Fällen handelt es sich um $d y$ nastische Königshäuser, die vor allem auf dem Lande und bei den Wüstenstämmen Unterstützung genießen. Angesichts der außenpolitischen Bedrohungslage von Jordanien durch die Konfliktachsen von Palästina über den Libanon (Hisbollah u. a.) und Syrien bis zum Iran ist dessen Lage als prekär zu bezeichnen. Hier sei nur an jüngste Rohstoffentwicklungen und -perspektiven der USA ab 2020 erinnert, denen zufolge sich die bisherigen Sicherheitskonzeptionen in Nahost (Schutzmacht für

15 In erweiterter Analyse müssten alle »arabischen« Länder für eine ausgeweitete Differentialdiagnose einbezogen werden, um eine Stichprobe nach der abhängigen Variable zu vermeiden.

16 In Jordanien soll ein Fünftel der Parlamentssitze nach jüngstem königlichen Dekret den Listen der bislang nicht oder deutlich unterrepräsentierten Gruppen geöffnet sein, vor allem den Palästinensern. Gleichzeitig wird die Quote für Frauen erhöht (Economist 2013 a). 
Saudi-Arabien und die Emirate und damit für den Status quo) dramatisch verschieben werden (Mills 2012). ${ }^{17}$

Auch der zweite Faktor: entfremdete Eliten im Dissens darüber, welcher Reformkurs eingeschlagen werden soll (in Richtung einer islamisch geprägten politischen Gemeinschaft oder einer eher westlich liberalen Demokratie unter Zurückdrängen der rückwärtsgewandten religiösen $\mathrm{Kräfte}^{18}$ ), ist in diesen sechs Ländern anzutreffen. Dabei geht es auch im ethnisch-kulturell eher homogenen Tunesien um den Übergang zu einer pluralistischen Demokratie muslimischer Prägung, was von der regierenden Al-Nahda-Partei befürwortet wird, oder zu einer säkularen Demokratie. „Wir wollen leben wie ihr in Europa«, so hieß eine der ursprünglichen Forderungen der dortigen Bürger (Lerch 2011: 42). Die Äußerung des tunesischen Staatspräsidenten (Marzouki 2012) zeugt eher von Mäßigung. Die gleiche grundsätzliche Weichenstellung stellt sich für Ägypten, wo sich zu Beginn des Jahres 2013 eine Dreier-Machtkonstellation ergibt, möglicherweise mit folgendem Ausgang:

»A plausible division of spoils and responsibility might give the Brotherhood the domains of governance dearest to it - education, social welfare, and the judiciary - with the military getting defense, intelligence, the peace with Israel, the military ties to the United States, and a retention of the officer corps' economic prerogatives. Liberal secularists would have large numbers, a say in the rhythm of daily life in a country so hard to regiment and organize, and the chance to field a compelling potential leader in a future presidential election« (Ajami 2012: 64).

In Libyen (wie im Jemen und anderswo) ist die Herausforderung nicht nur von dissentierenden Eliten geprägt, sondern auch durch ethnisch-kulturelle Spannungslinien bestimmt (siehe unten). Die Forderung der Sicherstellung der nationalen Bodenschätze oder eines gerechten Anteils an ihnen ist dabei zentral.

Die autokratischen Herrschaftshäuser einschließlich der Emirate und Saudi-Arabiens gründen ihre Herrschaft auf die Ausbeutung der Bodenschätze und eine daraus

17 Insgesamt erweist sich die Faktorenliste von Goldstone als erstaunlich treffsicher, mit Blick auf den Ablauf und die Folgen der Massenproteste aber weniger. Einige bedeutende Unterschiede seien in aller Kürze benannt: Die Effizienzkrise der als ungerecht wahrgenommen staatlichen Autorität ist für Marokko und Jordanien trotz der Herausforderungen nicht festzustellen, in allen anderen sechs Ländern aber deutlich. In beiden Ländern gilt die Formel »König und Vaterland « als Basis der Regimelegitimation.

18 Ein jüngstes Interview von Staatspräsident Mursi (2013) lässt ungeachtet der Aussage »Wir wollen keinen Gottesstaat« hier Ambivalenzen hinsichtlich der Verpflichtung durch Scharia und Verfassung erkennen. Die Beziehungen zu den Kopten (ca. 10\% der Bevölkerung) und anderen Religionen seien gut und würden durch ihn weiter gestärkt. 
bezahlte wohlfahrtsstaatliche Absicherung der Bevölkerung, gepaart mit einem repressiven Staatsapparat (Economist 2013 b), der Zustimmung der wahhabitischen Stämme bzw. der jeweiligen Dynastie in den Emiraten und der sunnitischen Religionsführer. Rivalisierende Stämme sind vorerst neutralisiert. Diese eher traditionale Form der Legitimität gerät nunmehr durch die Forderung eines Wandels in Richtung Zivilgesellschaft und offener Demokratie unter Anpassungsdruck. Momente traditionaler Legitimität sollen durch Formen expliziter Zustimmung an den Wahlurnen zumindest ergänzt werden. Auch der Herrscher von Katar sieht sich diesem Druck ausgesetzt. Seine eigene Palastgarde umfasst lediglich 1000 Mann, die Armee 8000 Soldaten bei einer Einwohnerzahl von zwei Millionen. Die beiden anderen Säulen seiner Herrschaft sind die amerikanische Truppenpräsenz mit der größten Militärbasis im Nahen Osten und der Erfolg des liberalen Fernsehsenders Al-Dschasira, der eine entscheidende Rolle für die Verbreitung der Massenanliegen und -proteste seit $2010 \mathrm{im}$ Nahen Osten spielt. Ähnlich wie bei den Herrschern in Saudi-Arabien mit ihrer Finanzierung konservativer und rückwärtsgewandter religiöser Bestrebungen wird der gleiche Weg monetärer und medialer Intervention beschritten. Dabei ist bezeichnend, wie schnell der alleinregierende Scheich von einer medialen Unterstützung des Mubarak-Regimes in Ägypten auf die Seite der Protestler umgeschwenkt ist. Die gewaltigen Gasvorräte haben den Kataris das höchste Pro-Kopf-Einkommen in der Welt mit über 100.000 Euro pro Jahr beschert, freilich deutlich ungleich verteilt.

Wenn sich eine Vorhersage treffen lässt, dann die, dass das sich öffnende Herrschaftsmodell in Katar eher mit demjenigen in Saudi-Arabien fallen wird. SaudiArabien ist der Schlüsselstaat, Katar demgegenüber fast eine Orchidee. Der Anspruch beider, zu einer regionalen Ordnungsmacht zu werden, ist in beiden Fällen überzogen: Die Saudis und Kataris haben das Geld, aber beide nicht die politische Botschaft, um stabile Mehrheiten entstehen zu lassen. Im Unterschied zum Vorbild der Türkei (z. B. für die Al-Nahda-Partei in Tunesien), bei allen fortbestehenden Feindschaften und Erinnerungen der Araber und Türken, ist die Legitimität der türkischen Führung keine geborgte und extern abgesicherte. In dieser Hinsicht ist die sich herausbildende neue Herrschaftsform in Ägypten vielleicht mit der Türkei zu vergleichen. Allerdings ist das wirtschaftliche Entwicklungsniveau deutlich unterschiedlich (BSP pro Kopf im Jahr 2011 in Ägypten: 2.801 US-Dollar; in der Türkei: 10.524 US-Dollar; im Iran zum Vergleich 6.977 US-Dollar). ${ }^{19}$ Eben das, was die

19 Basierend auf nominalen Daten der United Nations Statistics Division (http://unstats.un.org/). Die Werte in einheimischer Kaufkraft (purchasing power parity, PPP) liegen um etwa ein Viertel weniger weit auseinander. 
Türkei an relativer Autonomie, auch angesichts des fortbestehenden Kurdenproblems, entfalten kann, geht Ägypten ab, das nach wie vor auf Militär- und Wirtschaftshilfe aus den USA (im Jahr 2012 ca. 1,5 Milliarden US-Dollar, nur übertroffen von der Hilfe für Israel) und auf Kredite des IWF angewiesen ist (die Schuldenverhandlung allein mit dem IWF beläuft sich Mitte des Jahres 2013 auf fast 5 Milliarden US-Dollar) (The National 2013).

Marokko liegt geostrategisch geschützter und weit weg von den Konfliktherden im östlichen Mittelmeer und besitzt im Unterschied zu Jordanien (Phosphat, Pottasche und Ölschiefer) ebenfalls erhebliche Bodenschätze zur Stabilisierung der autoritären Herrschaft (Phosphat mit 70\% des Exportanteils sowie Kupfer, Salz, Erdöl, Gold, Silber, Mangan, Cobalt und Erdgas) und sollte angesichts dieser und anderer benannter Vorteile vor einem nachhaltigen Überschwappen der Protestwelle tendenziell eher geschützt sein.

In Bahrain (etwa $50 \%$ der Bevölkerung sind rechtlose zugewanderte Arbeitskräfte) ruft die schiitische Bevölkerung mit einem Anteil von drei Viertel an der Gesamtbevölkerung nach politischer und wirtschaftlicher Aufwertung, wogegen die sunnitischen Herrscher saudische und katarische Truppen zur Hilfe ins Land riefen. Der Jemen und Syrien mit seiner ethnisch-kulturellen Vielfalt (und des Fehlens von verteilbaren Reichtümern, die aus der Nutzung von Bodenschätzen stammen) gehören mit Blick auf gespaltene Eliten ebenfalls in eine Gruppe neben Libyen (siehe oben). Ein erster Befund aus dieser Analyse der acht Länder mit Rebellionserfahrungen lautet: Traditionale Herrschaftsformen mit noch wenig mobilisierten Bevölkerungen (Marokko, Saudi-Arabien und Jordanien) können umfassenden Regimeherausforderungen bislang entgehen, können diese aber nicht ausschließen und reagieren deshalb mit Konzessionen (angekündigte Verfassungsreformen), manchmal auch nur symbolischen (Economist 2013 b).

Weder garantiert der Reichtum durch Bodenschätze die Abwesenheit solcher Forderungen nach Systemöffnung, der Emanzipation der Frauen oder gar Systemveränderung (wenn auch bislang nur latent, siehe Saudi-Arabien), noch treten solche Forderungen bislang in derartigen Herrschaftsformen mit deutlich schlechteren wirtschaftlichen Bedingungen (Jordanien und Marokko) auf. Vor vorschnellen strukturellen Analyseergebnissen muss also gewarnt werden. Traditionale Herrschaftsformen, unabhängig davon, ob mit Bodenschätzen gepaart oder nicht, können das Entstehen von Massenprotesten bislang unterbinden oder eindämmen. In allen diesen Ländern haben die Parlamente wenig zu sagen (Economist 2013 b). Stattdessen gibt es traditionale Verfahren der Anhörung und Problemlösung durch die Scheichs, vor allem umfangreiche Wohlfahrtszahlungen. Reichliche staatliche Mittel können die Sprengwirkungen zunehmender sozio-ökonomischer Ungleichheit 
für eine Zeit lang überspielen oder einhegen. Eine rein traditionale Form der politischen Legitimität würde solche zunehmenden Spannungen nicht abfangen können.

Mit Mancur Olson (1963) ist zu bedenken, dass wirtschaftliches Wachstum Instabilität durch unterschiedliche Nachfrage nach bestimmten Produkten mit sich bringt, und ferner mit Simon Kuznets (1955), dass zunehmende Einkommens- und Vermögensungleichheit bei sich öffnenden und erweiternden Märkten zu gesellschaftlichem Sprengstoff werden kann. ${ }^{20}$ So kann ein wohlfahrtsstaatlich abgesicherter Paternalismus bei Überforderung durch eine zu stark wachsende und unzureichend ausgebildete Bevölkerung und ein steigendes Anspruchsniveau schnell an seine Grenzen gelangen. Dies gilt vor allem, wenn im Sinne von Huntington (1968) ein institutionalisiertes Parteiensystem mit flexiblen und neue Bevölkerungsgruppen integrierenden Parteien fehlt, kurzum wenn das entscheidende Kriterium der Demokratie fehlt: die inhaltlich offene Zukunft für die jetzigen und mögliche zukünftige Machthaber (Adam Przeworski et al. 2000).

Die Herrschaftsform in Libyen und Syrien muss dagegen als sultanistische Autokratie bezeichnet werden. Die Herrschaft ist allein auf den »Sultan« ausgerichtet, auf seinen Clan und seine ethnische Gruppe. Die Entwicklung machtbrechender institutioneller Mechanismen ist undenkbar. Dass dies in Ägypten nicht ganz der Fall war, sieht man an der in Teilen erstaunlichen Unabhängigkeit der Richter den alten und neuen Machthabern gegenüber. Dabei werden allerdings auch Machtpositionen aus der alten Herrschaftsform beim Übergang in eine stärker gewaltenteilende Herrschaftsform gesichert.

In allen sultanistischen Regimen des Nahen Ostens ist es zum Bürgerkrieg gekommen. Die höchsten Opferzahlen (Syrien: fast 100.000 Tote laut UN-Schätzungen; siehe unten) sind in diesen Ländern zu verzeichnen. Im Falle Bahrains wurde ein solcher Bürgerkrieg durch die Intervention von Truppen aus Saudi-Arabien und Katar im Kern erstickt (weniger als 50 Tote). Auch gab es nur Ankündigungen einer bescheidenen Erweiterung der Parlamentsrechte. Im Jemen kam es zu mehr als 2.000 Toten allein in diesen Ereignissen. Der Bürgerkrieg in Libyen schließlich konnte nur durch die militärische und finanzielle Hilfe von außen (Arabische Liga, Katar) und den Einsatz der NATO-Luftwaffe gewonnen werden.

Der dritte Faktor von Goldstone: massive Wohlfahrtsverluste (bzw. nur sehr geringe Zuwächse) in Stadt und Land lässt sich leicht mit Hilfe des BertelsmannTransformationsindex (BTI) und der dortigen Quellen belegen (Völkel 2012: 18):

20 Allerdings sorgt der »Tunnel-Effekt« von Albert Hirschman (1973) auch dafür, dass bei nach unten in der Sozialpyramide durchsickernden Geldern auch die später Bedienten eher geduldig sein werden. Bezugspunkt ist hier die Geduld bei den Hinteren in einer sich langsam bewegenden Warteschlange in einem Tunnel. 
Wieder fallen Marokko (4,5\% BSP-Zunahme pro Kopf und Jahr, 2008-2012) und Jordanien (2,6\%) als Abweichler auf. Für alle Länder gilt aber die dauerhafte Herausforderung der Massen durch drastische Preissteigerungen in den Grundnahrungsmitteln angesichts weltweiter Nachfrage.

Geht man zum vierten Faktor: dem Entstehen einer angriffswilligen elitegeleiteten Volkskoalition über, so fehlt auch dieses Moment in Jordanien und Marokko. In Tunesien erklärte sich die Armee nach den anfänglichen Massenprotesten schnell für neutral (bis sympathisierend mit den Aufständischen). Ähnliches gilt für Ägypten. In Libyen gab es zahlreiche Überläufer aus der Armee und den Polizeikräften. Entscheidend aber war die militärische Auseinandersetzung: Ernsthaft gekämpft haben von allen betroffenen Herrschern nur Muammar al-Gaddafi, der mit seinem Leben bezahlt hat, und Assad, der sich in seinen Hochburgen verschanzt.

In Tunesien sind im Verhältnis zur Bevölkerung (knapp 11 Millionen) die wenigsten Opfer zu verzeichnen, ist der Prozess des Regimewandels also relativ friedlich verlaufen. Unwesentlich höher ist der Anteil der Opfer in Ägypten mit über 91 Millionen Einwohnern und in Bahrain mit 1,2 Millionen Einwohnern. Die Turbulenzen in Ägypten sind Ende Dezember 2012 nach der Absegnung der neuen islamistischen Verfassung durch die Wähler vermutlich nicht abgeklungen (nur ein Drittel der Wahlberechtigten beteiligte sich). Die De-facto-Dreiteilung der Macht zwischen den Muslimbrüdern, der Armee und der Wirtschaft zeigt eine Möglichkeit der Einhegung verbreiteter Massenproteste auf.

EntscheidendisthieraberdasVerhaltenderinternenSicherheitskräfteundderArmee (Chorley 1943).Erklärensich diesefürneutralodersympathisierenin Teilen(Tunesien und Ägypten) mit einer Regimetransformation, bekommt die Koalition der Angreifer neuenSchub.ImnunmehrzweieinhalbjährigenBürgerkrieginSyrienistdiesbesonders deutlichzusehen, wodie anfangs friedlichenund dannschwachbewaffneten, abervielfachgespaltenenOppositionsgruppennichtnurPersonalundWaffenvonaußerhalbdes Landeserhalten,sondernauchdurchÜberläuferderArmeegestärktwerden,undseiesnur durch deren Exil in der Türkei. ${ }^{21}$

Auch bei dem fünften Faktor: eine zusammenschweißende, Angriffe rechtfertigende und Alternativen aufzeigende Ideologie, fallen die autokratischen Königreiche heraus, was nicht verwundert, sind doch die ersten drei Bedingungen nachhaltige Voraussetzung für das Vorliegen der fünften und letzten Bedingung.

21 Mehr als 50 Generäle haben nach Presseberichten demissioniert (siehe hierfür z. B. http:// www.yalibnan.com/2013/01/01/a-syrian-army-general-several-officers-defected-to-turkey/; 02.05.2013). 
Als Zwischenfazit ist festzuhalten, mit welch klaren Linien die hier angewandte und mitunter erweiterte Faktorenliste von Goldstone zur Erklärung beiträgt: Sie stellt den Konsens in der vergleichenden Revolutionsliteratur dar. Somit sind von den acht Ausgangsländern, in denen Massenmobilisierungen mit systemverändernden Forderungen anzutreffen waren, zwei als relativ stabil zu bezeichnen: Marokko und Jordanien. Aber auch hier gilt die Warnung von Huntington (1968), dass eine Reform von oben das Schwierigste überhaupt ist, muss sich der bisherige Herrscher doch gegen zwei Fronten, Konservative und Radikale, gleichermaßen behaupten. Man nimmt den bislang Privilegierten etwas weg und gibt es den Unterprivilegierten. Deren Forderungen oder die ihrer »Sprecher« gehen aber oft darüber hinaus. Grundlegende Reformen von oben sind historisch denn auch eher seltene Ereignisse (Meiji-Reform in Japan, Mustafa Kemal Atatürk in der Türkei).

Die anderen sechs Länder zerfallen in drei Gruppen: erstens die mit relativ friedlichem Übergang (Tunesien und Ägypten), zweitens den mittels externer militärischer Intervention zum vorläufigen »Erfolg « gebrachten Übergang in Libyen oder den abgewendeten Regimewandel in Bahrain und die nach wie vor offene Lage im Jemen (International Crisis Group 2012) mit den religiösen Spaltungen zwischen der Mehrheit der Sunniten und der Minderheit der schiitischen Zaiditen (einer religiösen Untergruppe) sowie der separatistischen Al-Huthi-Bewegung im Norden. Drittens steht Syrien allein da. Die Assad-Herrschaft hat ihre Überlebensfähigkeit nur noch der außen- und sicherheitspolitischen Balance und Interessenlage von Russland und China und vorrangig den USA zu verdanken.

All dies bedeutet aber nicht, dass aus Stammeskämpfen und ethnischem Separatismus, aus der Benachteiligung von ethnischen Gruppen, auch bei wirtschaftlichem Fortschritt, nicht bürgerkriegsähnliche Zustände in den anderen Ländern entstehen können. Die Überlappung der Konfliktformen von Stammesfehden, ethnischem Separatismus, Kleinkrieg lokaler Kriegsherren, Bürgerkriegen und externen Interventionen, internem und transnationalem Terrorismus ist im arabischen Raum ohnedies ein Dauerthema. Nimmt man die Differenzierungen der Revolutionstheorie auf, so sind viele der revolutionären Situationen keineswegs als abgeschlossene revolutionäre Veränderungen zu begreifen. In Ägypten spielen die alten Kräfte in der Justiz und der Armee nach wie vor eine zentrale Rolle, die jederzeit (z. B. Aufrollen des Mubarak-Prozesses) ein Eingreifen ermöglicht.

Man darf mit Charles Tilly (1978) schließlich nicht übersehen, dass die Kosten einer dauerhaften Massenmobilisierung nicht nur für die Wirtschaft insgesamt, sondern auch für die Individuen enorm hoch sind. Wer Mobilisierung sagt, benennt auch gleichzeitig das Abfallen der Mobilisierung, die Demobilisierung zu einem späteren Zeitpunkt, was neuen autoritären Herrschern und Versuchungen Tür und 
Tor öffnen kann. Allerdings sind sich Beobachter (Cohen 2012; Hottinger 2011) darin einig, dass die Grunderfahrung des Stürzens einer unbeliebten, brutalen, korrupten und ineffizienten Führung zu den unverzichtbaren Erfahrungen für die Zukunft gehören dürfte, mit Hottinger (2011), dass man seine Würde wiedergewonnen habe. Ob daraus demokratisch-westliche Vorstellungen von Demokratie mit ihrer Meinungsoffenheit, Gewaltenteilung und Gewaltfreiheit resultieren, bleibt abzuwarten.

\section{Optionen und Aussichten}

Grundlegend ist das Ordnungsproblem (Hobbes 1991). Das Gewaltmonopol ist in allen sechs Ländern gefährdet, nicht in den beiden Monarchien. Die Einhaltung des Gewaltmonopols ist grundlegend für zweierlei: einigermaßen stabile Bedingungen des Wirtschaftens und das Entstehen politischer Institutionen, die einen Gesellschaftspakt erlauben und ihre Wirkung unabhängig vom unmittelbaren Druck sozialer Kräfte (Huntington 1968) ausüben.

Was die aus beiden Bereichen zu gewinnende politische Legitimität anlangt, so ist die Theorie spezifischer und diffuser Unterstützung von David Easton (1975) grundlegend. Eine Herrschaftsform wird erst nach einiger Zeit, also mit wirtschaftlichen Erfolgen, auch Legitimität gewinnen, diffuse Unterstützung auch in Krisen gegenüber der spezifischen Unterstützung, die nur in guten Zeiten gewährt wird, also keinen Kreditpuffer darstellt. Erst diese wirtschaftliche Basiseffizienz wird den Freiraum für akzeptierte politisch-institutionelle Formen ermöglichen, möglicherweise auch für eine pluralistisch-säkulare Demokratie westlichen Zuschnitts, so unwahrscheinlich sie angesichts der geäußerten Präferenz der Massen auf dem Lande ist. In den Städten ergibt sich freilich ein anderes Bild der Zustimmung. ${ }^{22}$

Kern der politischen Beruhigung ist die Einigung zwischen Alt- und Neu-Eliten (Colomer 2000; Roland 2000), das Überleben der alten Eliten (Mubarak) und die Chance gescheiterter Eliten, bei den nächsten Wahlen zum Erfolg zu kommen. ${ }^{23}$ Herrscht Misstrauen vor, gepaart mit mangelnder politischer Institutionalisierung, so steigt die Versuchung der Externalisierung interner politischer Konflikte durch die Mobilisierung der jeweiligen Klientel, sei es gegen Israel oder gegen andere ethno-religiöse Gruppen. Edward Mansfield und Jack Snyder (2005; vgl. auch Zim-

22 Auch hier ist die Analyse von Stadt-Land-Differenzen im Zuge der Bildung politischer Ordnung durch Huntington (1968) grundlegend.

23 Roland (2000) hebt besonders die Fähigkeit politischer Führer hervor, aus den zwischenzeitlichen Verlierern neue Entschädigungskoalitionen in der jeweils nächsten Runde der politischen Auseinandersetzung zu bilden, um zur Stabilität eines neuen (demokratischen) Gemeinwesens beizutragen. 
mermann 2004) heben bei ihrer demokratiekritischen Sicht des Regimewandels gerade die Gefährdung von Demokratien in einem autokratischen Umfeld hervor. Dieser Effekt kommt zu den bestehenden Belastungen durch ethno-kulturelle Bande und Konflikte, durch Migration und Medieneinflüsse hinzu.

Selbst diese Dreiheit: Gewaltmonopol, wirtschaftliche Basiseffizienz und sich entwickelnder institutioneller Pluralismus unterliegt starken außenpolitischen Gefährdungen durch Irredenta, religiös-kulturelle Abspaltungen, Clan-Loyalitäten, Waffenlieferungen, fünfte Kolonnen und Terrorismus und angesichts des sich vermutlich bald wandelnden Sicherheitskonsenses in Nahost (Spiegel 2013).

Die Arabellion hat die Herrscher (nicht unbedingt die Herrschaftsstrukturen) in allen Fällen bis auf Bahrain und Syrien abgelöst. Doch in den Aussichten für die zweite und dritte Phase des Regimeübergangs sind über die bisher genannten Faktoren hinaus noch weitere prägende Unterschiede zwischen den Ländern zu berücksichtigen. Zur Vielzahl der bisherigen Einzelaspekte lassen sich in aller Kürze länder- und revolutionsvergleichend folgende prägende Unterschiede hinzufügen:

Tunesien, wo mit der Selbstverbrennung eines Gemüsehändlers am 17. Dezember 2010 in einer Provinzstadt die Protestwelle ihren Ausgang nahm, weist die größte ethnische Homogenität (eine weit über 90\%ige Identifikation mit den Arabern) auf, mit das höchste Niveau der Schulbildung (wenn auch weniger in Bezug auf Frauen), und nachLibyen(2009: 9.957 laufendeUS-Dollar) dashöchstePro-Kopf-Einkommen der Vergleichsstaaten (ca. 9000 US-Dollar; Algerien 4.567, Ägypten 2.698 und Syrien 2.893 US-Dollar). ${ }^{24}$ Der relativ gewaltfreie Übergang der Macht(ca. 340 Tote) aufdie islamische $\mathrm{Al}$-Nahda-Partei lässt - bei allen Unsicherheiten einer Prognose - die Entwicklungschancen dieses Landes vergleichsweise am besten erscheinen, wozu auch die Lage fernab von den nahöstlichen Konfliktherden und das französische »Erbe« beitragen. Die Koalition der Protestler umfasste vorwiegend den (kleinen) säkularen Mittelstand (Selbständige, Handwerker) und Freiberufler wie Anwälte, Ingenieure, Ärzte und Personen des öffentlichen Dienstes.

Nimmt man freilich das Nachbarland Algerien, wo vor 22 Jahren die Armee mit äußerster Härte gegen islamistische Strömungen im eigenen Lande vorgegangen ist (bis zu 150.000 Tote werden geschätzt; White o. J.), wo diese Armee das Regime ungebrochen stützt und wo nur wenige Konzessionen an örtliche Protestler gemacht worden sind, dann wird eine Prognose schon bedenklicher. Der algerische Dichter

24 In Bahrain liegt der Wert bei 18.184 US-Dollar, ist aber deutlich ungleicher verteilt. Die Weltbank liefert kein Verteilungsmaß für Bahrain (siehe http://data.worldbank.org/indicator/ NY.GDP.PCAP.CD; 02.05.2013). 
Boualem Sansal (2012) meint gar, in der algerischen, weiterhin repressiven Entwicklung den Ausgang der Rebellion in vielen arabischen Ländern zu sehen.

In Ägypten als dem größten Flächenstaat unter den Vergleichsländern hat sich die Armee ebenso wenig wie in Tunesien hinter den Machthaber gestellt, sondern ist neutral geblieben. Die Protestkoalition umfasste ähnlich wie in Tunesien Elemente des säkularen Mittelstandes, viele Jugendliche und erst in einer späteren Phase unterstützende Gruppen mit stärkerer religiöser Bindung. Hier sind die Gewinner der Wahlen (die Freiheits- und Gerechtigkeitspartei der Muslimbruderschaft und ihre Bündnispartner in der Demokratischen Allianz mit 47,2\% der Sitze im Jahr 2012) nicht die Träger der revolutionären Bewegung, sondern der bestorganisierte Wettbewerber (eben die Muslimbruderschaft), der in vielen Bereichen (Sicherheit, Schulen, Versorgung) öffentliche Güter für die Bevölkerung zu stellen vermag. Insgesamt erreichten die islamistischen Parteien mehr als zwei Drittel der Mandate. Auf dem Lande ist wie in allen Vergleichsländern die traditionale islamische Bindung ungleich stärker ausgeprägt als in den Städten. Die Bevölkerung ist deutlich inhomogener in Ägypten (ca. 90\% Sunniten und 10\% koptische Christen); die bisherigen deutlichen Gewinner im alten Regime, das Militär und die Großbourgeoisie, sehen sich zwar steigender Kritik ausgesetzt, dürften aber noch gut im System verschanzt sein. Das Ausmaß der Gewalt kann insgesamt als relativ gering angesehen werden (ca. 850 Tote).

Mit Libyen ${ }^{25}$ steigt sowohl das Ausmaß der Gewalt (mehr als 10.000 Tote) wie die Einmischung des Auslands ${ }^{26}$ von zwei Seiten, der Arabischen Liga mit ideologischer Unterstützung der Rebellen und mit Waffenlieferungen sowie mit dem Kampfeinsatz der NATO im UN-Auftrag. Die entscheidende Spaltungslinie sind hier die Stammesgrenzen zwischen Cyreneika (Sanoussi; Ölvorräte), Tripolitanien (mit zahlreichen Stämmen; Öl) und Fessan (Öl und andere Bodenschätze) im Süden mit einem hohen Berberanteil. Durch die massiven Kämpfe ist das Gewaltmonopol stärker als in den anderen Ländern (mit Ausnahme Syriens) geschwächt, alte und neue Rechnungen werden beglichen (z. B. Ermordung des übergelaufenen Rebellenanführers General Fattah Junis durch islamistische Gruppen). Die Bodenschätze des Landes bieten große Reichtumsreserven. Sie können aber auch gleichzeitig den Fluch des Rohstoffreichtums mit sich bringen, der im rent seeking (Vorteilsnahme durch Eroberung der Politik) kleiner Gruppen, in der Verzerrung der Preise und

25 Die dortigen Bewegungen stürmten Kasernen und Polizeistationen, bewaffneten sich und gingen in den Widerstand, ein gänzlich anderes Muster als in Tunesien oder Ägypten.

26 Jüngst wieder mit dem Eingreifen von Djihadisten in den syrischen Bürgerkrieg. 
einer inflationären Wirtschaft endet und es nicht schafft, die Ausbildung und damit die Produktivität des eigenen Volkes auf eine neue Stufe zu heben.

In Bahrain musste das sunnitische Herrscherhaus Militärhilfe aus dem ebenfalls sunnitischen Saudi-Arabien gegen die Forderungen der schiitischen Zwei-DrittelMehrheit anfordern, was für vorübergehende Ruhe gesorgt hat. Hier, ebenso wie in Saudi-Arabien, Marokko und den Emiraten, haben finanzielle Wohltaten der Herrscherhäuser die Massenunzufriedenheit vorerst besänftigt. Andererseits beziehen diese Länder und die anderen reichen Emirate einen Teil ihrer Legitimation nicht nur aus verteilten Ölgeldern, sondern auch aus traditionalen Herrschaftsmustern. Bei den Saudis und den reichen Emiraten ist dies die Stellung des Scheichs als Primus inter Pares unter den Beduinen.

Das Ausmaß der politischen Reformen in Marokko mit einer begrenzten Abgabe der königlichen Macht an das Parlament ist dabei größer als die Scheinzugeständnisse etwa in Jordanien. Insgesamt ist das politische System in allen Monarchien der arabischen Welt durch größere relative Festigkeit und klare Loyalität des Militärs und der Geheimdienste gekennzeichnet. Ein geläufiger Grund für die mangelnde Unterstützung der Herrscher durch die Armee in Tunesien, Ägypten und Libyen liegt in der Bevorzugung der eigenen Palastgarde und eigener Sicherheitsdienste gegenüber der Armee.

\section{Szenarien}

Wie aus der vergleichenden Revolutionsanalyse bekannt, sind die hauptsächlichen Träger des Protests selten die Gewinner der Neuerungen. Wer schließlich die wesentlichen Kosten der Modernisierung trägt, ist eine offene Frage. Die zentrale Rolle des Nichteingreifens der Armeekräfte (Chorley 1943) für einen Erfolg der revolutionären Herausforderer bestätigt sich auch hier, ebenso wie die der Unterstützung durch externe Mächte. Auch gilt die Lehre weiterhin, dass eine Gruppe allein Protest bedeuten kann, eine revolutionäre Herausforderung aber immer eine breite Protestkoalition über diverse Soziallagen hinweg verlangt. Allerdings scheinen diese Protestkoalitionen eher uneins oder deutlich gespalten, ob eine Lebensform wie in Europa, wie in der Türkei (Vorbild für die Al-Nahda-Partei in Tunesien, weniger in Ägypten, das deutliche Vorbehalte gegen eine osmanische Bevormundung hat) oder eine (schrittweise oder versteckte) Wiedereinführung der Scharia $^{27}$ (wie auch in der Türkei in mancher Hinsicht) dominieren soll.

27 Eine solche Forderung wirft die Frage auf, ob der geistige Unterbau für Pluralismus und Individualismus nicht schwächelt, vor allem weil Anhänger der Scharia mit ihrer umfassenden Regelung diese beiden geradezu bekämpfen. 
Auf jeden Fall spielen Faktorausstattung (Finanz- und Humankapital, Boden und Arbeit), geographische Lage und religiös-kulturelle Spannungslinien nach wie vor eine zentrale Rolle bei den Entwicklungschancen wie vor allem auch die in der vergleichenden Institutionenökonomie (North 1992; Buzan 2004; Acemoglu/Robinson 2012) hervorgehobene Ausdifferenzierung zentraler Institutionen. Diese sollen Sicherheit geben und damit die Transaktionskosten täglichen Wirtschaftens senken. Gerade aus dieser Sicht erklärt sich ja der Erfolg der Partei der Muslimbrüder in Ägypten.

Auch wird deutlich, dass Gewalt bei revolutionären Umsturzversuchen kein zentraler Faktor ist oder sein muss. Die Zahl der Gewaltopfer in dieser Arabellion ist vergleichsweise gering, mit Ausnahme Libyens und Syriens und auch des Jemen, wo der Präsident schließlich zur Machtübergabe an seinen Stellvertreter ${ }^{28}$ bereit war, um den Bürgerkrieg nicht noch weiter anzuheizen.

Die Bedeutung einer der zentralen Erklärungsursachen für Revolutionen - externe Niederlagen der Armee und damit deren grundsätzliche Schwächung als Stabilisator der Macht - findet sich in all diesen Fällen eher in den internen Bereich und auf die Spannungslinien zu den Sicherheitsgruppen und Geheimdiensten verlagert. Andererseits ist mit der Aufblähung des Militärhaushalts zur Sicherung der eigenen Herrschaft, angesichts des Palästinakonflikts und der Rivalität zwischen der Türkei und ihren Nachbarn, zwischen Saudi-Arabien und Iran unter anderem ein starker Ressourcenanteil der normalen Wohlfahrt entzogen. Die eigentlich auf den Schutz der eigenen Grenzen gerichtete professionelle Armee wird in den Interessenkonflikten innerhalb des eigenen Landes tendenziell geschwächt oder neutralisiert sich selbst.

Geht man von den theoretischen Einsichten der vergleichenden Revolutionsforschung aus, ergänzt um diejenigen für das Entstehen interner Ordnung und wirtschaftlicher Entwicklung sowie internationaler Stabilität und Kooperation, so bieten sich mindestens fünf grundlegende turbulente Szenarien, vier davon gewaltsam, an: erstens das Zerriebenwerden zwischen den Extremen, beispielsweise im Falle des bislang schon nicht mehr so moderaten Ministerpräsidenten Mursi (Economist 2013 d) durch scharia-orientierte und gewaltbereite Gruppen wie ungebremste Forderungen nach einem säkularen Staat.

Die zweite Variante, welche Folge der ersten sein, aber auch ohne diese zustande kommen kann, ist der Staatszerfall. Systemübergreifend ergibt sich eine klare Ab-

28 Diese Variante der Machtübergabe an einen der engsten Vertrauten des ehemaligen Machthabers ist nach Linz und Stepan (1996) ein Spezifikum des Sultanismus. Oft handelt es sich auch nur um eine Machtübernahme: Der Neue kennt die Spielregeln des alten Systems am besten. 
folge für die Schritte zur Stabilisierung eines Regimes, und im Umkehrschluss für dessen Zerfall: Das Wichtigste ist die Einrichtung und Verteidigung des staatlichen Gewaltmonopols nach innen (Polizei) und außen (Armee). Ohne alltägliche Sicherheit wird sich niemals auch nur das geringste Ausmaß an Loyalität der Bevölkerung gewinnen lassen. Blickt man auf den institutionellen Rahmen und die wirtschaftliche Effizienz eines neuen Regimes, so bietet sich eine einfache Theorie mit großer Reichweite an. Verblüffend ist, wie wenig sie Gemeingut der Herrschenden zu sein scheint: Easton (1975) spricht von einem (demokratischen) Schönwettersystem. Solange die wirtschaftlichen Erfolge des Systems auch bei der Bevölkerung ankommen, ist diese bereit, das System zu unterstützen. Bleibt der Erfolg aus, wird diese spezifische, erfolgsabhängige Unterstützung nicht mehr gewährt. Die Kosten der Herrschaft steigen dramatisch. Erst nach einer langen Schönwetterperiode tritt der Fall ein, dass die Unterstützung unabhängig von einer Leistungsverschlechterung, also einer Krise, gewährt wird. Diffuse Unterstützung, Kredit wird dann gewährt. Diese Theorie ist hochgradig erklärungskräftig. Sie erklärt das Scheitern der Weimarer Republik, den Erfolg der alten Bundesrepublik und die großen Reserven gegenüber der Demokratie in Ostdeutschland. Je weniger diffus also die Unterstützung ist, desto prekärer werden Machtverschiebungen zu neuen Eliten. Kulturelle und religiöse Eigenarten können diese grundsätzlichen Zusammenhänge noch weiter verschärfen. So ist etwa der Bereich der Religion einzigartig darin, dass er private Werte mit dem Bereich der Politik und öffentlichen Werten und zugleich Vorstellungen über das Jenseits zu verknüpfen hilft (Richardson 2007). Dies ist eine nahezu unerschöpfliche Mischung des Dissens und öffentlichen Unbehagens. Unter Bedingungen des Staatszerfalls ist dann auch leicht mit sezessionistischen Bewegungen wie in Libyen, Syrien und möglicherweise Jordanien zu rechnen. Religiöse Momente waren in der Initialphase dieser Rebellionen nahezu unbedeutend. Es ging um den angehäuften Verdruss über wirtschaftliches Versagen, Korruption und Unfähigkeit der herrschenden Eliten. Nach deren Sturz treten religiös-gebundene oder sich religiös orientierende politische Führer ${ }^{29}$ auf den Plan, um ihre Herrschaftsund Gesellschaftsmodelle durchzusetzen, sehen sich aber weiteren, deutlich säkular, freilich uneinig (Economist 2013 d) geprägten Protesten gegen solche Bestrebungen ausgesetzt.

Die Außenpolitik bestimmt das dritte Szenario, sind doch - vielleicht mit Ausnahme Ägyptens - alle diese Länder zu klein für eine autonome Entwicklung oder zu schwach, ihre Autonomie nach außen zu verteidigen. Ein militärischer Konflikt

29 Beispiele sind Mursi in Ägypen oder Hamadi Jebali in Tunesien, der im Februar 2013 nach der Ermordung des Oppositionsführers Chokri Belaid zurückgetreten ist. 
zwischen Israel und dem Iran ließe viele bisherige Fronten durcheinandergeraten und würde im arabischen und iranischen Protest alle religiösen Gräben überwinden. Ferner sind ethnische Minderheiten oft von hohem instrumentellem Wert für externe Patrone. Angesichts der Verschlechterung der israelisch-türkischen Beziehungen sagte mir einmal ein hoher israelischer Sicherheitsfachmann: »There are Kurds everywhere «.${ }^{30}$ Drei Flanken bieten sich also für eine fortdauernde Destabilisierung an: erstens externe Kriege, zweitens Sezessionsversuche und drittens Ausnutzen ethnischer und religiöser Spannungen. Hier geht es dann nicht nur um die »bloody boundaries« (ein vielgescholtener Terminus von Huntington; 1996), sondern auch um »blurred boundaries«, verwischte Grenzen.

Das vierte Szenario nimmt seinen Ausgang vom Zerfall Syriens. Syrien gemahnt nach dem zweijährigen blutigen Bürgerkrieg und der Zerstörung vieler Städte an das Schicksal Somalias (Economist 2013 c). Oppositionsgruppen, rivalisierende Warlords, Islamisten und Gewaltbanden, mit Unterstützung aus den Nachbarländern operierend und viele offene Rechnungen präsentierend, beschwören einen Nahost-Flächenbrand herauf. ${ }^{31}$ Die Rede ist von einem möglichen globalen jihad, sollten die chemischen und atomaren Waffen außer Kontrolle geraten und eingesetzt werden. Präsident Assad hat durch den Einsatz staatlicher Gewalt gegen die vorwiegend von Sunniten getragenen Proteste die Macht seiner Unterstützer, der Alawiten, erhalten können, aber inzwischen auch bei seinen Verbündeten im Ausland (Russland und China) nahezu jegliche Unterstützung verloren, was sich allerdings in der Jahresmitte 2013 zu ändern scheint.

Ein gänzlich anderes - fünftes - Szenario böte eine innere Reform in den islamischen Religionen, ein Rückbesinnen auf tolerante Traditionen und auch auf die Notwendigkeit, ihrer Glaubensgemeinschaft hierarchische Strukturen einzuziehen, wenn das Funktionieren des politischen Gemeinwesens nicht dauerhaft auf dem Spiel stehen soll, wenn Kontrolle der radikalen Varianten religiöser Ausprägung und nicht gar deren fortdauernde Instrumentalisierung durch Geistliche erreicht werden soll. Sollten sich die Türkei als vorwiegend sunnitischer muslimischer Staat und Indonesien als vorwiegend sunnitischer Staat, aber ohne eine muslimische Staatsreligion, zu erfolgreichen Staatsmodellen für Ordnung, Modernisierung und Liberalisierung entwickeln, so gewönnen diese nicht nur unmittelbar an Macht, sondern könnten zu den regionalen Ordnungsmächten heranwachsen, deren Fehlen Huntington (1996) beklagt hat.

30 Informelles Gespräch im Jahr 2006 in Ankara.

31 So unterstützt die Hisbollah aus dem Libanon als schiitische Partei und Miliz Assad gegen die große Mehrheit der Sunniten, kurdische Milizen operieren im Nordosten Syriens. 
Die größte Hoffnung ergibt sich schließlich aus konzilianteren Führungen, wie in Südafrika oder in Nordirland, aus Führern, die angesichts des Leerlaufens bisheriger Kampfstrategien auf Versöhnung und Einvernehmen setzen, die Gefolgschaft ihres eigenen Lagers sicherstellen und somit das Monopol der Staatsgewalt bei diesen grundlegenden Veränderungen nicht gefährden. Ein solches Szenario speist sich aus der Erfahrung, durch Gewaltgebrauch und Reformunfähigkeit weltweit in der eigenen Entwicklung noch stärker zurückzufallen, als dies schon in den Zeiten vor der nachhaltig globalisierten Wirtschaft der Fall war. Es ist mit Sicherheit kein Zufall, dass mit dem rasanten Aufstieg der Republik Irland, damit demjenigen der katholischen Iren insgesamt, eine Versöhnungsstrategie zwischen Protestanten des Nordens und beiden katholischen Elementen im Norden und Süden eingesetzt hat. Zuvor schon hatten Frederik de Klerk und Nelson Mandela mit dem Zusammenbruch des kommunistischen Blocks und externer militärischer Unterstützung die vorteilhaftere Lage einer offenen Gesellschaft mit großen Exportchancen (und eben keinem Exportboykott) klar vor Augen. Mandela hat auch bei der (nord-)irischen Konfliktmediation mitgewirkt (Moltmann 2007).

Eine solche Kalkulation rationaler Wahl steht vielen Politikern in Nahost sicherlich vor Augen. Oft rückt sie aber angesichts kurzfristiger (gewaltsamer) Gewinne in den Hintergrund, wenn nicht gar an ihrem großen Entwurf gezweifelt wird. An Dynamik würde sie alles überschatten und den Nahen Osten zu einem der schärfsten weltweiten Wettbewerber innerhalb von ein bis zwei Generationen werden lassen können. Eine solche Vision bekäme ebenfalls einen gewaltigen Schub durch eine größere israelische Konzession. Eine andere völlig neue Entwicklung käme zustande durch einen massiven Einstieg der Chinesen in die dortige Wirtschaft und Infrastruktur. Angesichts des gewaltigen Rohstoffbedarfs Chinas ist vermutlich eher mit einer solchen Entwicklung als mit der einer größeren israelischen Konzession (auch in symbolischer Hinsicht mit Blick auf Jerusalem) zu rechnen.

Keineswegs sind hiermit schon alle wichtigen Szenarien hinreichend angedeutet. Internationale Migrationswellen (unter anderem von Sunniten aus Syrien in den Irak und Libanon und nach Jordanien, wo überall die jetzt schon prekäre Austarierung religiös-kultureller Gruppen ins Rutschen gerät), die Weiterverbreitung chemischer, atomarer und anderer Waffen in einer Region ohne jegliches staatliches und (bis auf Israel) regionales Gewaltmonopol können leicht zur größten internationalen Herausforderung seit dem Zweiten Weltkrieg werden. Hinzu kommen die durch die neue Rohstoffpolitik der USA deutlich veränderten dortigen Sicherheitsbelange, weniger die der Europäer mit ihrer geringen militärischen und auch finanziellen 
Kapazität zur Eindämmung eskalierender Konfliktherde. ${ }^{32} \gg$ Es gibt Dekaden, in denen nichts passiert; und es gibt Wochen, in denen sich Dekaden abspielen«, so lässt sich die Erfahrung manchen Revolutionärs zusammenfassen. Nach allem, auch den wenig überraschenden Massenprotesten gegen eine westliche Provokation, darf man im arabischen Raum mit seiner kulturellen und religiösen Vielfalt auf Überraschungen gefasst sein.

\section{Schlussbemerkungen}

Man kann die Frage aufwerfen, warum die hier in den Vordergrund gerückten Bausteine und nicht andere Theorien gewählt worden sind. In aller Kürze lässt sich dazu sagen: Erstens sollte die gewählte Analyse möglichst für sich sprechen. Ohne Zweifel erweist sich die vergleichende Revolutionsanalyse in ihrer Anwendung auf die arabischen Rebellionen als ergiebig. Nicht nur Fehlklassifikationen wie arabische "Revolution « lassen sich mit guten Gründen zurückweisen, wie auch allgemein der komplexe Verlauf bisheriger Revolutionen zu größter Vorsicht gegenüber Vorhersagen und Extrapolationen mahnt. Auch ist die Lage im arabischen Raum von einer derartigen Komplexität und Unsicherheit gezeichnet, dass Prognostiker ähnlich wie mit den Entwicklungen mit dem Zusammenbruch des europäischen Kommunismus nach 1989 leicht auf dem falschen Fuß erwischt werden und wurden.

Zweitens erweist sich die Kombination mit der Transformationslehre politischer Systeme, vor allem ihrer Pfadabhängigkeit, als geeignet, das weitverbreitete sultanistische Erbe in einer ersten Gesamtsicht angemessen zu deuten. ${ }^{33}$ Die Feinheiten der politischen Transformationslehre in ihren vielen Ausgestaltungen verlieren sich oft in deskriptiven Elementen und hätten hier auch den Platz gesprengt. In den meisten Fällen sind ja erst minimale Schritte der institutionellen politischen Transformation gegangen, sodass ein Erfahrungsvergleich mit anderen Transformationen voreilig wäre.

Im Rückgriff auf die vergleichende Revolutionsanalyse lassen sich nicht nur Krisen und große Systemherausforderungen angemessen einordnen. Der Blick wird

32 Die Neuorientierung der amerikanischen Rohstoffpolitik (z. B. durch den »fracking«-Abbau von Ölschiefer und Gas), die Chance, selbst zum Großexporteur von Rohstoffen zu werden, wird innerhalb von 10 Jahren vermutlich alle beschriebenen und weitere Szenarien entscheidend beeinflussen.

33 Im Übrigen sollte klar geworden sein, dass man mit einer »These« oder nur einem Aspekt wie dem des Sultanismus vieles andere zugleich vernachlässigt. Hier und allgemein in fruchtbaren empirischen Forschungen mittlerer Reichweite wird einem aus den Erklärungserfolgen und trotz allem dem Prinzip einer gewissen Erklärungsökonomie folgenden theoretischen Eklektizismus gehuldigt, wie er bei einer so jungen Disziplin wie der vergleichenden Politikwissenschaft unvermeidlich ist. 
auch auf zahlreiche differentia specifica in den einzelnen Konfliktländern gerichtet. Nimmt man die Differenzierungen der Revolutionstheorie auf, sind viele der revolutionären Situationen keineswegs als abgeschlossene revolutionäre Veränderungen zu begreifen. Theorien aus der vergleichenden Regimeanalyse richten sich auf $R e$ gimealternativen. So scheidet ein ähnlich wie in der Vergangenheit gewandetes autokratisches Regime in Tunesien, Ägypten, Syrien und letztlich überall im arabischen Raum aus. Cohen (2012) nennt das Bewusstsein, nach Dekaden schlimmster Unterdrückung und Rückständigkeit erfolgreich rebelliert zu haben, eine historische Kernerfahrung, ein historisches Plebiszit mit Langzeitwirkung. Aber autoritäre Regime können in religiös-kulturellem Wandel, durch externe Wirtschaftsauflagen und durch die Verwicklungen in externe und innere Kriege, schnell heraufbeschworen werden. So sehr totalitäre oder post-totalitäre Regime keine Alternative zu autokratischen Lösungen darstellen, so unsicher bleibt die Prognose, ob der Weg zu einer freiheitlich-toleranten politischen Ordnung in diesen Ländern gefunden wird. Demokratien werden durch Demokraten, durch immer neue Kompromisse gemacht (Linz 1998), und solche Kompromissbereitschaft ist allgemein eher gegeben, wenn die Wirtschaftslage nicht zusätzliche Belastungen mit sich bringt.

Einmal mehr gilt Huntingtons (1968) Diagnose, dass sich Schwierigkeiten nicht durch arme Länder ergeben, sondern durch solche, die reich werden wollen. Der Weg zur Modernität ist allemal mit Krisen gepflastert. Die weltweite Verteilung der Güter und Fähigkeiten ist kein Nullsummenspiel, aber durch enorme Bestrafungen und Verwerfungen für zurückfallende Länder geprägt.

Rein strukturalistische Gesichtspunkte wie Bevölkerungszusammensetzung und -wachstum, Alphabetisierung (Elsayyad/Hanafi 2013), Beteiligung der Frauen, Freiheitsrechte und Wirtschaftseffizienz, wie sie z. B. nicht immer schlüssig im Bertelsmann-Transformationsindex (BTI) zu finden sind, wenden leicht den Blick von der Handlungsebene (vgl. Hussain/Howard 2013). ${ }^{34}$ Hier sind es Individuen, noch mehr Gruppen, die sich mobilisieren und dies nur angesichts sonst noch höherer Opportunitätskosten. Die Mobilisierung in einem autokratischen Regime ist für das Individuum gefährlich, die Offenbarung der persönlichen Meinung gegenüber einer in der Öffentlichkeit anders bekundeten (Kuran 1995) immer ein großes Risiko. Hier lassen sich keine Vorhersagen über den individuellen Mut von Dissidenten in autokratischen Regimen machen. Deshalb muss der Prognoseversuch auf der Basis von BTI-Entwicklungen auch eher scheitern (Völkel 2012). Die »Sicher-

34 Weder die Qualität der jeweils angewandten Skalen noch die statistisch sehr einfachen Weiterverarbeitungen der Rohdaten werden hinreichend reflektiert. Diskriminante Validierungen finden sich ebenso wenig in diesen Studien wie robuste ökonometrische Spezifikationen. 
heit in der Zahl der Massenprotestierer«, die Mobilisierungskaskaden und die damit erreichte Dynamik, noch verschärft durch neue Formen der Handy- und Internetkommunikation und Organisation haben zweierlei gezeigt: die Schwierigkeit einer Prognose $^{35}$ und die verringerten Chancen des Gegensteuerns einer völlig überraschten staatlichen Autorität. Dabei ist nicht nur die schnelle Mobilisierung der Protestler erstaunlich, sondern auch der rasche Wechsel von friedlichen Protestgruppen zu Kampfgruppen des Stadtguerillatyps (Noll 2013). ${ }^{36}$ Dieser Wechsel traf die Herrschenden urplötzlich und stellt möglicherweise einen zentralen Typ gewaltsamer Auseinandersetzung in der Zukunft dar.

Eine vergleichende Analyse der »Arabellion « kann die Revolutionsanalyse in mehrfacher Hinsicht bereichern: Zum einen kann sich die Zahl erfolgreicher wie auch gescheiterter Revolutionen (etwa durch militärische Niederlage in einem Bürgerkrieg oder einen militärischen Gegencoup) vergrößern, was wichtige Einsichten für die Differentialdiagnose liefert. Zum anderen werden die Auswirkungen »neuer « Formen der Kommunikation und Mobilisierung unter den Rebellierenden und der Zwang des Reagierens oder der Unfähigkeit zur Antwort unter ihren Gegnern breiter erfasst werden können. Auch Momente politischer Trägheit, passiver Hinnahme von Herrschaftsformen, gegen die man sich eigentlich aufgelehnt hat, werden festzustellen sein. Auf lineare Entwicklungen zu setzen, ist vermutlich naiv. Wiederkehrende Elemente größerer Freiheiten, die dann wieder von denselben oder anderen Akteuren eingeschränkt werden, kurzum: die jeweilige politische Gelegenheitsstruktur bei Wahlen, im Schatten anderer wichtiger (nationaler) und internationaler Ereignisse und Herausforderungen wird Überraschungen mit sich bringen, die skeptisch gegenüber allzu »sicheren« Prognosen machen sollten. Nur eines wird deutlich: Im Sinne der Regimetransformationslehre, vor allem der in ihrem Gefolge oft erfolgenden wirtschaftlichen Transformation, ist für längere Zeit - einfach auf Grund der Unordnung des Alten, Gestürzten und der fehlenden und akzeptierten Neuordnung - mit einem Niedergang und dann vielleicht erst mit einer allmählichen Besserung zu rechnen. ${ }^{37}$

35 Angesichts der vielfältigen Aggregatsformen menschlichen Handelns auf der Makroebene staatlichen Handelns darf man hier eher skeptisch bleiben.

36 Noll (2013) beschwört außerdem eine rasant wachsende internationale gewaltintensive islamistische Front.

37 Ein J-Kurvenmuster - hier nicht zu verwechseln mit der J-Kurve von James Davies (1962) - als mögliche Erklärung für die Entstehung von Revolutionen. 


\section{Literatur}

Acemoglu, Daron/Robinson, James A. 2012: Why Nations Fail: The Origins of Power, Prosperity, and Poverty, New York, NY.

Ajami, Fouad 2012: The Arab Spring at One: A Year of Living Dangerously, in: Foreign Affairs 91: 2, 56-65.

Barber, Benjamin 2012: Brennende Botschaften, in: Süddeutsche Zeitung, 18.09.2012, 2.

Bredekamp, Horst 2012: An der Front des Bilderweltbürgerkrieges, in: Süddeutsche Zeitung, 19.09.2012, 11.

Bueno de Mesquita, Bruce/Smith, Alastair/Siverson, Randolph M./Morrow, James D. 2003: The Logic of Political Survival, Cambridge, MA.

Bussmann, Margit/Hasenclever, Andreas/Schneider, Gerald (Hrsg.) 2009: Identität, Institutionen und Ökonomie. Ursachen innenpolitischer Gewalt, Politische Vierteljahresschrift, Sonderheft Nr. 43, Wiesbaden.

Buzan, Barry 2004: From International to World Society? English School Theory and the Social Structure of Globalisation, Cambridge, MA.

Chorley, Katherine 1943: Armies and the Art of Revolution, London.

Cohen, Roger 2012: This is a Revolution, in: New York Times International Weekly, 26.11.2012.

Colomer, Josep 2000: Strategic Transitions, Baltimore, MD.

Davies, James C. 1962: Toward a Theory of Revolution, in: American Sociological Review 27: 1, 5-19.

Easton, David 1975: A Re-assessment of the Concept of Political Support, in: British Journal of Political Science 5: 4, 435-457.

Economist 2013 a: Jordan's Election: Bad for the King, 19.01.2013, 36-37.

Economist 2013 b: Repression in the Gulf: A Mirage of Rights, 19.01.2013, 37.

Economist 2013 c: Syria: The Death of a Country, 23.02.2013, 11, 20-21.

Economist 2013 d: Briefing Egypt, 02.02.2013, 10-11, 17-19.

Elsayyad, May/Hanafy, Shima'a 2013: Voting Islamist or Voting Secular? An Empirical Analysis of Voting Outcomes in »Arab Spring « Egypt, Zürich.

Freedom House 2011: Freedom in the World 2011, Washington, D.C.

Goldstone, Jack A. 2003: Comparative Historical Analysis and Knowledge Accumulation in the Study of Revolutions, in: Mahoney, James/Rueschemeyer, Dietrich (Hrsg.): Comparative Historical Analysis in the Social Sciences, Cambridge, 41-90.

Hibbs, Douglas A. 1973: Mass Political Violence: A Cross-National Causal Analysis, New York, NY. 
Hirschman, Albert O. 1973: The Changing Tolerance for Income Inequality in the Course of Economic Development, in: Quarterly Journal of Economics 87: 4, 544-566.

Hobbes, Thomas 1991: Leviathan. Herausgegeben von Richard Tuck, Cambridge. Hodler, Roland 2013: The Political Economics of the Arab Spring, Zürich.

Hottinger, Arnold 2011: Arabische Umbrüche - erste Runde. Wo steht die arabische Revolution?, in: http://www.journal21.ch; 31.08.2011.

Huntington, Samuel P. 1968: Political Order in Changing Societies, New Haven, CT.

Huntington, Samuel P. 1984: Will More Countries Become Democratic?, in: Political Science Quarterly 99: 2, 193-218.

Huntington, Samuel P. 1991: The Third Wave: Democratization in the Late Twentieth Century, Norman, OK.

Huntington, Samuel P. 1996: The Clash of Civilizations and the Remaking of World Order, New York, NY.

Hussain, Muzammil M./Howard, Philip N. 2013: What Best Explains Successful Protest Cascades? ICTs and the Fuzzy Causes of the Arab Spring, in: International Studies Review 15: 1, 48-66.

International Crisis Group 2012: Yemen: Enduring Conflicts, Threatened Transition (Middle East Report No. 125), Sanaa.

Kennedy, Paul 1989: The Rise and Fall of the Great Powers: Economic Change and Military Conflict from 1500 to 2000, New York, NY.

Kuran, Timur 1995: The Inevitability of Future Revolutionary Surprises, in: American Journal of Sociology 100: 5, 1528-1551.

Kuznets, Simon 1955: Economic Growth and Income Inequality, in: American Economic Review 45: 1, 1-28.

Landes, David S. 1998: The Wealth and Poverty of Nations: Why Some are So Rich and Some So Poor, New York, NY.

Lefebvre, Georges 1970: La Grande Peur de 1789, Paris.

Lerch, Wolfgang Günter 2011: Die arabische Revolution, in: Die politische Meinung Nr. 498, 42-47.

Linz, Juan J. 1998: Democracy's Time Constraints, in: International Political Science Review 19: 1, 19-37.

Linz, Juan J./Stepan, Alfred 1996: Problems of Democratic Transition and Consolidation: Southern Europe, South America, and Post-Communist Europe, Baltimore, MD.

Lipset, Seymour Martin 1994: The Social Requisites of Democracy Revisited, in: American Sociological Review 59: 1, 1-22. 
Mansfield, Edward D./Snyder, Jack 2005: Electing To Fight: Why Emerging Democracies Go To War, Cambridge, MA.

Marzourki, Moncef 2012: The Arab Spring Still Blooms, in: http://www.nytimes. com/2012/09/28/opinion/the-arab-spring-still-blooms.html?_r=0; 27.09.2012.

Merkel, Wolfgang 2010: Systemtransformation. Eine Einführung in die Theorie und Empirie der Transformationsforschung, 2. Auflage, Wiesbaden.

Mills, Mark P. 2012: Unleashing the North American Energy Colossus: Hydrocarbons Can Fuel Growth and Prosperity (Power \& Growth Initiative Report No. 1), New York, NY.

Moltmann, Bernhard 2007: Die Außenwelt der Innenwelt. Die externe Dimension einer Konfliktgeschichte (HSFK-Report Nr. 11), Frankfurt a. M.

Mursi, Muhammad 2013: Wir wollen keinen Gottesstaat. Interview, in: Frankfurter Allgemeine Zeitung, 19.01.2013, 5.

Noll, Chaim 2013: Islamischer Imperialismus. Neueste Entwicklungen im Nahen Osten, in: Merkur 67: 1, 78-84.

North, Douglass 1992: Institutionen, institutioneller Wandel und Wirtschaftsleistung, Tübingen.

Olson, Mancur 1963: Rapid Growth as a Destabilizing Force, in: Journal of Economic History 23: 4, 529-552.

Przeworski, Adam/Alvarez, Michael E./Cheibub, Jose Antonio/Limongi, Fernando 2000: Democracy and Development, New York, NY.

Reed, Stanley 2013: Qatar Spends to Propel its Global Agenda, in: International Herald Tribune, 07.01.2013, 1.

Richardson, Louise 2007: Was Terroristen wollen, Frankfurt a. M.

Roland, Gérard 2000: Transition and Economics: Politics, Markets, and Firms, Cambridge, MA.

Russett, Bruce M./Oneal, John R. 2001: Triangulating Peace: Democracy, Interdependence, and International Organizations, New York, NY.

Sansal, Boualem 2012: Es kann in einem religiösen Staat keine Demokratie geben. Gespräch mit dem Schriftsteller, in: DIE ZEIT, 06.10.2012.

Spiegel 2013: Geheime BND-Studie. Amerikas Öl verändert die Welt, in: http:// www.spiegel.de/politik/ausland/bnd-studie-zu-oelvorkommen-der-usa-a-8781 57.html; 17.01.2013.

Struwwel, Peter 1848: Handbüchlein für Wühler oder kurzgefasste Anleitung in wenigen Tagen ein Volksmann zu werden, 2. Auflage, Leipzig. 
The National 2013: ElBaradei Says Egypt's Government Must Compromise to Get»Vital«IMFLoan,in:www.thenational.ae/news/world/middle-east/elbaradei -says-egypts-government-must-compromise-to-get-vital-imf-loan;02.05.2013. Tilly, Charles 1978: From Mobilization to Revolution, Reading, MA. Tilly, Charles 1975: Revolutions and Collective Violence, in: Greenstein, Fred I./ Polsby, Nelson W. (Hrsg): Handbook of Political Science, 3. Auflage, Reading, MA, 483-555.

Trotzki, Leo 1967: Geschichte der russischen Revolution, Berlin.

Völkel, Jan 2012: The BTI 2012: Looking Back on the Arab Spring: An Interpretation of Recent Political Developments, in: Ghali, Amine/Hegazy, Ibrahim/ Kawakibi, Salam/Kienle, Eberhard/Manea, Elham/Saadawi, Samir/Schumacher, Tobias/Völkel, Jan (Hrsg.): The Arab Spring: One Year After. Transformation Dynamics, Prospects for Democratization and the Future of Arab-European Cooperation (Europe in Dialogue 2012/02), Gütersloh, 13-36.

Walt, Stephen M. 1996: Revolution and War, Ithaca, NY.

Weber, Max 1964: Wirtschaft und Gesellschaft (Studienausgabe), Köln.

Weiffen, Brigitte 2009: Entstehungsbedingungen von Demokratien, Baden-Baden. White, Matthew o. J.: Mid-Range Wars and Atrocities of the Twentieth Century, in: http://users.erols.com/mwhite28/warstat4.htm; 02.05.2013.

Zimmermann, Ekkart 1981: Krisen, Staatsstreiche und Revolutionen, Wiesbaden.

Zimmermann, Ekkart 2003: Vergleichende Krisen- und Konfliktforschung, in: Berg-Schlosser, Dirk/Müller-Rommel, Ferdinand (Hrsg.): Vergleichende Politikwissenschaft. Ein einführendes Handbuch, 4. Auflage, Opladen, 297-328.

Zimmermann, Ekkart 2004: Vergleichende Konfliktforschung. Demokratisierung und Externe Konflikte, in: Pfetsch, Frank (Hrsg.): Band 48 der Heidelberger Jahrbücher »Konflikt «, Heidelberg, 19-30.

Zimmermann, Ekkart 2006: Revolution, in: Heun, Werner/Honecker, Martin/Morlok, Martin/Wieland, Joachim (Hrsg.): Evangelisches Staatslexikon, 4. Auflage, Stuttgart, 2045-2049.

Zimmermann, Ekkart 2009: Formen des politischen Terrorismus. Ein Plädoyer für eine Differentialdiagnose, in: Vierteljahrshefte zur Wirtschaftsforschung 78: 4, 11-28.

Zimmermann, Ekkart [1983] 2011: Political Violence, Crises, and Revolutions: Theories and Research, New York, NY.

Zimmermann, Ekkart 2011: Globalization and Terrorism, in: European Journal of Political Economy 27: Supplement 1, 152-162. 
Zimmermann, Ekkart 2012: Makrogewalt: Rebellion, Revolution, Krieg, Genozid, in: Albrecht, Günter/Groenemeyer, Axel (Hrsg.): Handbuch soziale Probleme, 2. Auflage, Wiesbaden, 861-885.

\section{Der Autor}

Prof. Dr. Ekkart Zimmermann ist em. Professor für Makrosoziologie an der Technischen Universität Dresden.

E-Mail: EkkartZimmermann@t-online.de

Das Manuskript wurde im Juni 2013 abgeschlossen. 